ARGONNE NATIONAL LABORATORY

9700 South Cass Avenue

Argonne, Illinois 60439

ANL/MCS-TM-273

\title{
Benchmarking Optimization Software with COPS 3.0*
}

\author{
by \\ Elizabeth D. Dolan, ${ }^{\dagger}$ Jorge J. Moré, ${ }^{\ddagger}$ and Todd S. Munson ${ }^{\ddagger}$
}

Mathematics and Computer Science Division

Technical Memorandum ANL/MCS-TM-273

February 2004

\footnotetext{
${ }^{*}$ This work was supported by the Mathematical, Information, and Computational Sciences Division subprogram of the Office of Advanced Scientific Computing Research, Office of Science, U.S. Department of Energy, under Contract W-31-109-Eng-38, and by the National Science Foundation Grant DMI-0322580.

${ }^{\dagger}$ Department of Industrial Engineering and Management Sciences, Northwestern University, and Mathematics and Computer Science Division, Argonne National Laboratory, Argonne, IL 60439. dolan@mcs. anl.gov.

${ }^{\ddagger}$ Mathematics and Computer Science Division, Argonne National Laboratory, Argonne, IL 60439. \{tmunson, more $@$ @mcs . anl.gov
} 
Argonne National Laboratory, a U.S. Department of Energy Office of Science laboratory, is operated by The University of Chicago under Contract W-31-109-ENG-38.

\section{DISCLAIMER}

This report was prepared as an account of work sponsored by an agency of the United States Government. Neither the United States Government nor any agency thereof, nor The University of Chicago, nor any of their employees or officers, makes any warranty, express or implied, or assumes any legal liability or reponsibility for the accuracy, completeness, or usefulness of any information, apparatus, product, or process disclosed, or represents that its use would not infringe privately owned rights. Reference herein to any specific commericial product, process, or service by trade name, trademark, manufacturer, or otherwise, does not necessarily consittute or imply its endorsement, recommendation, or favoring by the United States Government or any agency thereof. The views and opinions of document authors expressed herein do not necessarily state or reflect those of the United States Government or any agency thereof.

Available electronically at http://www.osti.gov/bridge/

Available for a processing fee to the U.S. Dept. of Energy and its contractors, in paper, from:

U.S. Department of Energy

Office of Scientific and Techdnical Information

P.O. Box 62

Oak Ridge, TN 37831-0062

phone: (865) 576-8401

fax: (865) 576-5728

email: reports@adonis.osti.gov 


\section{Contents}

Abstract

Introduction 1

Testing Methods 1

1 Largest Small Polygon $\quad 3$

2 Distribution of Electrons on a Sphere 5

$\begin{array}{lll}3 & \text { Shape Optimization of a Cam } & 7\end{array}$

4 Hanging Chain $\quad 9$

5 Isometrization of $\alpha$-pinene $\quad 11$

6 Marine Population Dynamics $\quad 13$

$\begin{array}{llr}7 & \text { Flow in a Channel } & 16\end{array}$

8 Robot Arm $r$

9 Particle Steering $\quad 21$

10 Goddard Rocket $\quad 23$

11 Hang Glider $\quad 26$

12 Catalytic Cracking of Gas Oil 29

13 Methanol to Hydrocarbons 31

14 Catalyst Mixing 33

15 Elastic-Plastic Torsion $\quad 35$

$\begin{array}{ll}16 \text { Journal Bearing } & 37\end{array}$

17 Minimal Surface with Obstacle $\quad 39$

18 Triangular Mesh Smoothing $\quad 41$

19 Tetrahedral Mesh Smoothing $\quad 44$

20 Transition States for the Lane-Emden Problem $\quad 47$

21 Transition States for the Dirichlet Problem 49 
References 


\title{
Benchmarking Optimization Software with COPS 3.0
}

\author{
by
}

Elizabeth D. Dolan, Jorge J. Moré, and Todd S. Munson

\begin{abstract}
We describe version 3.0 of the COPS set of nonlinearly constrained optimization problems. We have added new problems, as well as streamlined and improved most of the problems. We also provide a comparison of the FILTER, KNITRO, LOQO, MINOS, and SNOPT solvers on these problems.
\end{abstract}

\section{Introduction}

The COPS [12] test set provides a modest selection of difficult nonlinearly constrained optimization problems from applications in optimal design, fluid dynamics, parameter estimation, and optimal control, among others. In this report we describe version 3.0 of the COPS problems. The formulation and discretization of the original problems have been streamlined and improved. We have also added new problems.

For each problem we discuss the formulation of the problem. We also present the structural data in the table below in order to provide an approximate idea of the size and sparsity of the problem.

\begin{tabular}{l} 
Variables \\
Constraints \\
Bounds \\
Linear equality constraints \\
Linear inequality constraints \\
Nonlinear equality constraints \\
Nonlinear inequality constraints \\
Nonzeros in $\nabla^{2} f(x)$ \\
Nonzeros in $c^{\prime}(x)$ \\
\hline
\end{tabular}

We include the results of computational experiments with the FILTER [13], KNITRO [35], LOQO [33], MINOS [25], and SNOPT [17] solvers. As part of the benchmarking process we have introduced an analyzer to help determine whether the quality of the solution returned by any particular solver meets our expectations. The analyzer will be described in a separate report. We have also introduced software scripts specifically designed to benchmark this test set.

\section{Testing Methods}

We have devised a set of Perl scripts for running a problem on each solver successively, so as to minimize the effect of fluctuation in the machine load. The scripts track the wall-clock time from the start of the solve, killing any process that runs for more than 1800 seconds, 
which we declare unsuccessful. We cycle through all problem variants, recording the wallclock time as well as the combination of AMPL system time (to interpret the model and compute varying amounts of derivative information required by each solver) and solver time. We consider the times returned by AMPL definitive, but we initially record the wall-clock times to check for discrepancies in the solvers' methods of calculating execution time. We include no problem results for which the AMPL time and the wall-clock time differ by more than ten percent. To further ensure consistency, we have verified that the AMPL time results we present could be reproduced to within five percent accuracy.

We examine the solver result number returned by each AMPL solver, but a successful return code is only the first step we take to check on the solver's work. As each solve completes, we run the analyzer to check the solution written. If the feasibility and optimality tests fail to meet our standards, we tighten whatever tolerance option applies for that solver by an order of magnitude and rerun the job. If the tolerance reaches 1.0e-16 and the solution reported does not meet our goals, then the solver fails the benchmark test, and we use the symbol $\uparrow$ in the appropriate table. We also check the solution returned by the AMPL solver when an unsuccessful return code is reported. These cases are marked with the symbol $\dagger$ in the appropriate table. Sometimes the benchmark test indicates that a solution has been obtained to within our tolerances.

Regarding solver options, we also increase iteration, super-basics, and memory size limits that might artificially cause a solver to fail. Printed output is reduced to its lowest level for each solver. The testing method described is more strict than anything we have done in the past, but the results we obtained from default options varied widely in quality. We felt that some independent measurement, while its specifics might be argued, would enhance the worth of the benchmark as a whole.

All computations were performed on an Intel Pentium $41.8 \mathrm{GHz} \mathrm{CPU}$ with $512 \mathrm{M}$ of RAM and a $256 \mathrm{~Kb}$ cache, running Red Hat 7.3. The tested solvers include

filterSQP, ASL (20020923)

KNITRO 3.0, ASL (20020905)

LOQO 6.02, ASL (20020221)

MINOS 5.5, ASL (20020614)

SNOPT 6.1, ASL (20020614)

The source code for all the problems in this report and for the analyzer is included with the distribution [12] of COPS 3.0. 


\section{Largest Small Polygon}

Find the polygon of maximal area, among polygons with $n_{v}$ sides and diameter $d \leq 1$.

\section{Formulation}

This is a classic problem (see, for example, Graham [19]). If $\left(r_{i}, \theta_{i}\right)$ are the coordinates of the vertices of the polygon, then we must maximize

$$
f(r, \theta)=\frac{1}{2} \sum_{i=1}^{n_{v}-1} r_{i+1} r_{i} \sin \left(\theta_{i+1}-\theta_{i}\right)
$$

subject to the constraints

$$
\begin{aligned}
r_{i}^{2}+r_{j}^{2}-2 r_{i} r_{j} \cos \left(\theta_{i}-\theta_{j}\right) \leq 1, & 1 \leq i<n_{v}, \quad i<j \leq n_{v} \\
\theta_{i} \leq \theta_{i+1}, & 1 \leq i<n_{v}, \\
\theta_{i} \in[0, \pi], r_{i} \geq 0, & 1 \leq i \leq n_{v} .
\end{aligned}
$$

Our implementation follows [16] and fixes the last vertex by setting $r_{n_{v}}=0$ and $\theta_{n_{v}}=\pi$. By fixing a vertex at the origin, we can add the bounds $r_{i} \leq 1$.

Graham [19] showed that the optimal solution is regular for odd $n$ but not regular for even $n$ except $n=4$. Another interesting feature of this problem is the presence of order $n_{v}^{2}$ nonlinear nonconvex inequality constraints. We also note that as $n_{v} \rightarrow \infty$, we expect the maximal area to converge to the area of a unit-diameter circle, $\pi / 4 \approx 0.7854$. This problem has many local minima. For example, for $n_{v}=4$ a square with sides of length $1 / \sqrt{2}$ and an equilateral triangle with another vertex added at distance 1 away from a fixed vertex are both global solutions with optimal value $f=\frac{1}{2}$. Indeed, the number of local minima is at least $O\left(n_{v} !\right)$. Thus, general solvers are usually expected to find only local solutions. Data for this problem appears in Table 1.1 .

Table 1.1: Largest-small polygon problem data

\begin{tabular}{|l|c|}
\hline Variables & $2\left(n_{v}-1\right)$ \\
Constraints & $\left(\frac{1}{2} n_{v}+1\right)\left(n_{v}-1\right)-1$ \\
Bounds & $2\left(n_{v}-1\right)$ \\
Linear equality constraints & 0 \\
Linear inequality constraints & $n_{v}-2$ \\
Nonlinear equality constraints & 0 \\
Nonlinear inequality constraints & $\frac{1}{2} n_{v}\left(n_{v}-1\right)$ \\
Nonzeros in $\nabla^{2} f(x)$ & $11\left(n_{v}-1\right)-8$ \\
Nonzeros in $c^{\prime}(x)$ & $2 n_{v}\left(n_{v}-1\right)-2$ \\
\hline
\end{tabular}

\section{Performance}

Results for the AMPL implementation are summarized in Table 1.2. A polygon with almost equal sides is the starting point. Global solutions for several $n_{v}$ are shown in Figure 1.1. 
Table 1.2: Performance on largest small polygon problem

\begin{tabular}{|c|c|c|c|}
\hline Solver & $n_{v}=50$ & $n_{v}=100$ & $n_{v}=200$ \\
\hline \hline FILTER & $27.64 \mathrm{~s}$ & $555.2 \mathrm{~s}$ & $\ddagger$ \\
$f$ & $7.66131 \mathrm{e}-01$ & $7.77239 \mathrm{e}-01$ & $\ddagger$ \\
$c$ violation & $8.88 \mathrm{e}-16$ & $1.17 \mathrm{e}-14$ & $\ddagger$ \\
optimality & $8.96 \mathrm{e}-07$ & $9.90 \mathrm{e}-07$ & $\ddagger$ \\
\hline KNITRO & $1.41 \mathrm{~s}$ & $8.99 \mathrm{~s}$ & $59.53 \mathrm{~s}$ \\
$f$ & $7.60725 \mathrm{e}-01$ & $7.37119 \mathrm{e}-01$ & $6.74980 \mathrm{e}-01$ \\
$c$ violation & $0.00 \mathrm{e}+00$ & $0.00 \mathrm{e}+00$ & $0.00 \mathrm{e}+00$ \\
optimality & $7.53 \mathrm{e}-07$ & $3.99 \mathrm{e}-07$ & $2.01 \mathrm{e}-07$ \\
\hline LOQO & $14.39 \mathrm{~s}$ & $\ddagger$ & $\ddagger$ \\
$f$ & $7.63694 \mathrm{e}-01$ & $\ddagger$ & $\ddagger$ \\
$c$ violation & $1.08 \mathrm{e}-10$ & $\ddagger$ & $\ddagger$ \\
optimality & $1.02 \mathrm{e}-10$ & $\ddagger$ & $\ddagger$ \\
\hline MINOS & $5.6 \mathrm{~s}$ & $121.3 \mathrm{~s}$ & $223.94 \mathrm{~s}$ \\
$f$ & $7.66297 \mathrm{e}-01$ & $6.79085 \mathrm{e}-01$ & $6.57163 \mathrm{e}-01 \dagger$ \\
$c$ violation & $8.03 \mathrm{e}-14$ & $1.75 \mathrm{e}-13$ & $2.66 \mathrm{e}-15 \dagger$ \\
optimality & $6.32 \mathrm{e}-08$ & $9.50 \mathrm{e}-10$ & $9.55 \mathrm{e}-02 \dagger$ \\
\hline SNOPT & $4.34 \mathrm{~s}$ & $69.35 \mathrm{~s}$ & $\ddagger$ \\
$f$ & $7.84015 \mathrm{e}-01$ & $7.85023 \mathrm{e}-01$ & $\ddagger$ \\
$c$ violation & $1.11 \mathrm{e}-10$ & $1.78 \mathrm{e}-11$ & $\ddagger$ \\
optimality & $8.30 \mathrm{e}-07$ & $1.35 \mathrm{e}-07$ & $\ddagger$ \\
\hline
\end{tabular}

$\dagger$ Errors. $\ddagger$ Timed out. $\uparrow$ Failed benchmark tests.
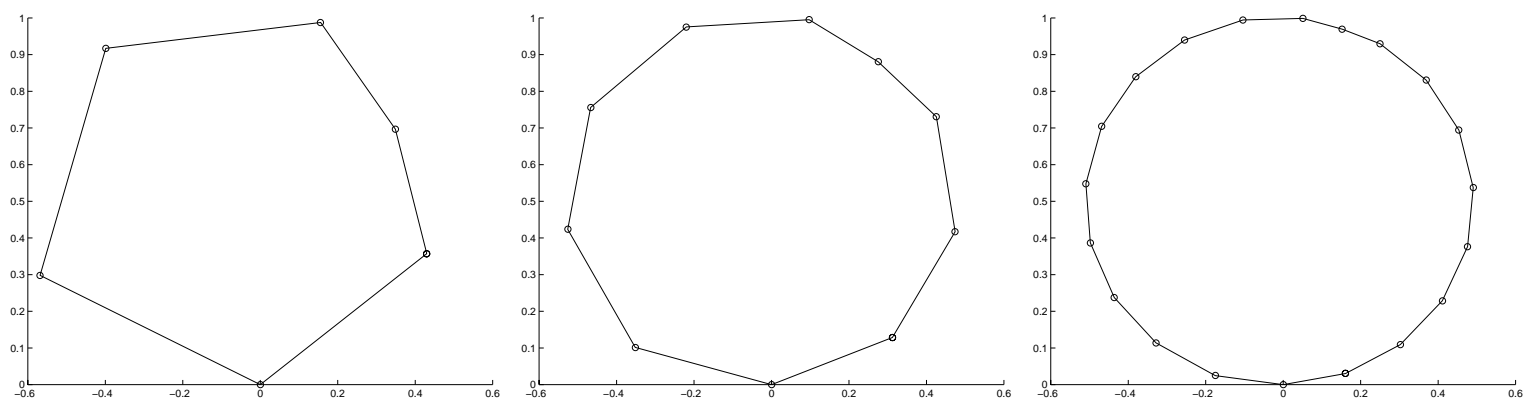

Figure 1.1: Polygons of maximal area with $n_{v}=6,10,20$ (left, center, right) 


\section{Distribution of Electrons on a Sphere}

Given $n_{p}$ electrons, find the equilibrium state distribution (of minimal Coulomb potential) of the electrons positioned on a conducting sphere.

\section{Formulation}

This problem, known as the Thomson problem of finding the lowest energy configuration of $n_{p}$ point charges on a conducting sphere, originated with Thomson's plum pudding model of the atomic nucleus. This problem is representative of an important class of problems in physics and chemistry that determine a structure with respect to atomic positions.

The potential energy for $n_{p}$ points $\left(x_{i}, y_{i}, z_{i}\right)$ is defined by

$$
f(x, y, z)=\sum_{i=1}^{n_{p}-1} \sum_{j=i+1}^{n_{p}}\left(\left(x_{i}-x_{j}\right)^{2}+\left(y_{i}-y_{j}\right)^{2}+\left(z_{i}-z_{j}\right)^{2}\right)^{-\frac{1}{2}}
$$

and the constraints on the $n_{p}$ points are

$$
x_{i}^{2}+y_{i}^{2}+z_{i}^{2}=1, \quad i=1, \ldots, n_{p} .
$$

Data for this problem appears in Table 2.1.

This problem has many local minima at which the objective value is relatively close to the objective value at the global minimum. Experimental and theoretical results $[24,27]$ show that

$$
\min \left\{f\left(v_{1}, \ldots, v_{n_{p}}\right):\left\|v_{i}\right\|=1,1 \leq i \leq n_{p}\right\} \geq \frac{1}{2} n_{p}^{2}(1-\varepsilon), \quad 0 \leq \varepsilon \leq\left(\frac{1}{n_{p}}\right)^{1 / 2} .
$$

Also, the number of local minima grows exponentially with $n_{p}$. Thus, determining the global minimum is computationally difficult, and solvers are usually expected to find only a local minimum.

Table 2.1: Electrons on a sphere problem data

\begin{tabular}{|l|c|}
\hline Variables & $3 n_{p}$ \\
Constraints & $n_{p}$ \\
Bounds & 0 \\
Linear equality constraints & 0 \\
Linear inequality constraints & 0 \\
Nonlinear equality constraints & $n_{p}$ \\
Nonlinear inequality constraints & 0 \\
Nonzeros in $\nabla^{2} f(x)$ & $9 n_{p}^{2}$ \\
Nonzeros in $c^{\prime}(x)$ & $3 n_{p}$ \\
\hline
\end{tabular}

\section{Performance}

Results for the AMPL implementation are summarized in Table 2.2. The starting point is a quasi-uniform distribution of the points on a unit sphere. The best known solution for $n_{p}=100$ is shown in Figure 2.1. 
Table 2.2: Performance on electrons on a sphere problem

\begin{tabular}{|c|c|c|c|}
\hline Solver & $n_{p}=50$ & $n_{p}=100$ & $n_{p}=200$ \\
\hline \hline FILTER & $34.51 \mathrm{~s}$ & $203.82 \mathrm{~s}$ & $\ddagger$ \\
$f$ & $1.05518 \mathrm{e}+03$ & $4.44841 \mathrm{e}+03$ & $\ddagger$ \\
$c$ violation & $5.55 \mathrm{e}-16$ & $1.22 \mathrm{e}-15$ & $\ddagger$ \\
optimality & $6.08 \mathrm{e}-07$ & $9.04 \mathrm{e}-07$ & $\ddagger$ \\
\hline KNITRO & $0.54 \mathrm{~s}$ & $3.12 \mathrm{~s}$ & $20.76 \mathrm{~s}$ \\
$f$ & $1.05518 \mathrm{e}+03$ & $4.44847 \mathrm{e}+03$ & $1.84390 \mathrm{e}+04$ \\
$c$ violation & $1.11 \mathrm{e}-16$ & $2.22 \mathrm{e}-16$ & $1.66 \mathrm{e}-16$ \\
optimality & $5.89 \mathrm{e}-08$ & $4.32 \mathrm{e}-08$ & $3.94 \mathrm{e}-07$ \\
\hline LOQO & $7.78 \mathrm{~s}$ & $\ddagger$ & $\ddagger$ \\
$f$ & $1.05518 \mathrm{e}+03$ & $\ddagger$ & $\ddagger$ \\
$c$ violation & $2.77 \mathrm{e}-08$ & $\ddagger$ & $\ddagger$ \\
optimality & $6.35 \mathrm{e}-07$ & $\ddagger$ & $\ddagger$ \\
\hline MINOS & $4.71 \mathrm{~s}$ & $\ddagger$ & $182.39 \mathrm{~s}$ \\
$f$ & $1.05518 \mathrm{e}+03$ & $\ddagger$ & $8.52026 \mathrm{e}+03 \dagger$ \\
$c$ violation & $5.25 \mathrm{e}-14$ & $\ddagger$ & $1.00 \mathrm{e}+00 \dagger$ \\
optimality & $1.62 \mathrm{e}-07$ & $\ddagger$ & $1.00 \mathrm{e}+00 \dagger$ \\
\hline SNOPT & $1.31 \mathrm{~s}$ & $10.42 \mathrm{~s}$ & $103.1 \mathrm{~s}$ \\
$f$ & $1.05518 \mathrm{e}+03$ & $4.44847 \mathrm{e}+03$ & $1.84389 \mathrm{e}+04$ \\
$c$ violation & $1.47 \mathrm{e}-13$ & $1.48 \mathrm{e}-13$ & $1.49 \mathrm{e}-13$ \\
optimality & $5.85 \mathrm{e}-07$ & $5.97 \mathrm{e}-07$ & $1.11 \mathrm{e}-07$ \\
\hline
\end{tabular}

$\dagger$ Errors. $\$$ Timed out. $\uparrow$ Failed benchmark tests.

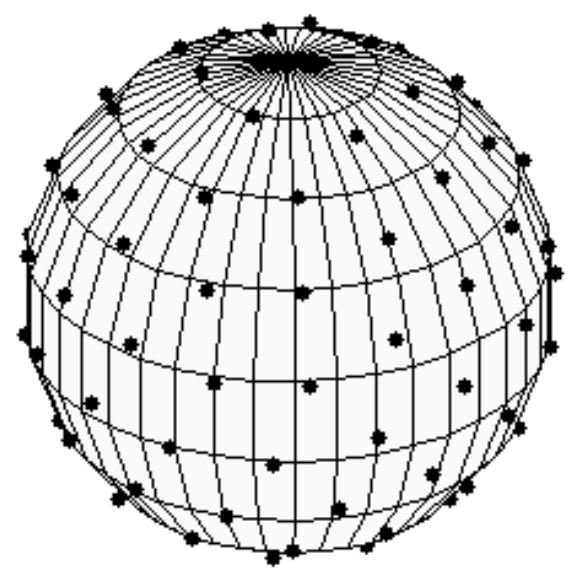

Figure 2.1: Optimal distribution of electrons on a sphere, $n_{p}=100$ 


\section{Shape Optimization of a Cam}

Maximize the area of the valve opening for one rotation of a convex cam with constraints on the curvature and on the radius of the cam.

\section{Formulation}

The formulation of this problem is due to Anitescu and Serban [1]. We assume that the shape of the cam is circular over an angle of $\frac{6}{5} \pi$ of its circumference, with radius $r_{\min }$. The design variables $r_{i}, i=1, \ldots, n$, represent the radius of the cam at equally spaced angles distributed over an angle of $\frac{2}{5} \pi$. We maximize the area of the valve opening by maximizing

$$
f(r)=\pi r_{v}^{2}\left(\frac{1}{n} \sum_{i=1}^{n} r_{i}\right)
$$

subject to the constraints on $r$. The design parameter $r_{v}$ is related to the geometry of the valve. We also require that $r_{\min } \leq r_{i} \leq r_{\max }$. The requirement that the cam be convex is expressed by requiring that

$$
\operatorname{area}\left(r_{i-1}, r_{i+1}\right) \leq \operatorname{area}\left(r_{i-1}, r_{i}\right)+\operatorname{area}\left(r_{i}, r_{i+1}\right),
$$

where area $\left(r_{i}, r_{j}\right)$ is the area of the triangle defined by the origin and the points $r_{i}$ and $r_{j}$ on the cam surface. This convexity constraint can also be expressed as

$$
2 r_{i-1} r_{i+1} \cos (\theta) \leq r_{i}\left(r_{i-1}+r_{i+1}\right), \quad i=0, \ldots, n+1,
$$

where $r_{-1}=r_{0}=r_{\min }, r_{n+1}=r_{\max }, r_{n+2}=r_{n}$ and $\theta=2 \pi / 5(n+1)$. The curvature requirement is expressed by

$$
-\alpha \leq\left(\frac{r_{i+1}-r_{i}}{\theta}\right) \leq \alpha, \quad i=0, \ldots, n
$$

This is a departure from [1], where the curvature constraint was expressed in terms of $\left(r_{i+1}-r_{i}\right)^{2}$. Data for this problem appears in Table 3.1 .

Table 3.1: Optimal design of a cam problem data

\begin{tabular}{|l|c|}
\hline Variables & $n$ \\
Constraints & $2 n$ \\
Bounds & $n$ \\
Linear equality constraints & 0 \\
Linear inequality constraints & $n-1$ \\
Nonlinear equality constraints & 0 \\
Nonlinear inequality constraints & $n+1$ \\
Nonzeros in $\nabla^{2} f(x)$ & 0 \\
Nonzeros in $c^{\prime}(x)$ & $5 n-3$ \\
\hline
\end{tabular}

We follow [1] and use $r_{\min }=1.0$ and $r_{\max }=2.0$ for the bounds on $r, r_{v}=1.0$ in the area of the valve, and $\alpha=1.5$ in the curvature constraint. Since the optimal cam shape is symmetric, we consider only half of the design angle. The problem was originally [1] formulated for the full angle of $\frac{4}{5} \pi$. 


\section{Performance}

Results for the AMPL implementation are summarized in Table 3.2. We use a starting guess of $r_{i} \equiv\left(r_{\min }+r_{\max }\right) / 2$. The cam shape for $\alpha=1.5$ appears in Figure 3.1. We note that the number of active constraints increases with $\alpha$ up to a threshold of $\alpha_{1} \approx 3.0$, after which increasing $\alpha$ does not change the optimal solution.

Table 3.2: Performance on optimal cam shape problem

\begin{tabular}{|c|c|c|c|}
\hline Solver & $n=800$ & $n=1000$ & $n=1200$ \\
\hline \hline FILTER & $2.47 \mathrm{~s}$ & $3.84 \mathrm{~s}$ & $5.71 \mathrm{~s}$ \\
$f$ & $4.27427 \mathrm{e}+00$ & $4.27399 \mathrm{e}+00$ & $4.27380 \mathrm{e}+00$ \\
$c$ violation & $8.88 \mathrm{e}-16$ & $1.33 \mathrm{e}-15$ & $8.88 \mathrm{e}-16$ \\
optimality & $1.08 \mathrm{e}-12$ & $1.46 \mathrm{e}-12$ & $2.09 \mathrm{e}-12$ \\
\hline KNITRO & $8.91 \mathrm{~s}$ & $14.87 \mathrm{~s}$ & $21.13 \mathrm{~s}$ \\
$f$ & $4.27427 \mathrm{e}+00$ & $4.27397 \mathrm{e}+00$ & $4.27380 \mathrm{e}+00$ \\
$c$ violation & $0.00 \mathrm{e}+00$ & $0.00 \mathrm{e}+00$ & $0.00 \mathrm{e}+00$ \\
optimality & $1.58 \mathrm{e}-10$ & $1.23 \mathrm{e}-12$ & $5.27 \mathrm{e}-12$ \\
\hline LOQO & $5.11 \mathrm{~s}$ & $53.19 \mathrm{~s}$ & $3.86 \mathrm{~s}$ \\
$f$ & $4.27427 \mathrm{e}+00$ & $4.27399 \mathrm{e}+00$ & $4.27380 \mathrm{e}+00$ \\
$c$ violation & $6.57 \mathrm{e}-14$ & $1.68 \mathrm{e}-14$ & $9.85 \mathrm{e}-14$ \\
optimality & $1.08 \mathrm{e}-12$ & $1.68 \mathrm{e}-12$ & $6.45 \mathrm{e}-10$ \\
\hline MINOS & $2.32 \mathrm{~s}$ & $3.17 \mathrm{~s}$ & $4.21 \mathrm{~s}$ \\
$f$ & $4.27427 \mathrm{e}+00$ & $4.27399 \mathrm{e}+00 \dagger$ & $4.27380 \mathrm{e}+00$ \\
$c$ violation & $2.28 \mathrm{e}-10$ & $1.33 \mathrm{e}-15 \dagger$ & $1.33 \mathrm{e}-15$ \\
optimality & $1.08 \mathrm{e}-12$ & $3.14 \mathrm{e}-03 \dagger$ & $6.16 \mathrm{e}-10$ \\
\hline SNOPT & $5.29 \mathrm{~s}$ & $8.71 \mathrm{~s}$ & $11.91 \mathrm{~s}$ \\
$f$ & $4.27427 \mathrm{e}+00$ & $4.27399 \mathrm{e}+00$ & $4.27380 \mathrm{e}+00$ \\
$c$ violation & $1.79 \mathrm{e}-15$ & $9.16 \mathrm{e}-12$ & $2.97 \mathrm{e}-13$ \\
optimality & $4.00 \mathrm{e}-11$ & $1.23 \mathrm{e}-12$ & $4.11 \mathrm{e}-11$ \\
\hline
\end{tabular}

$\dagger$ Errors. + Timed out. $\uparrow$ Failed benchmark tests.

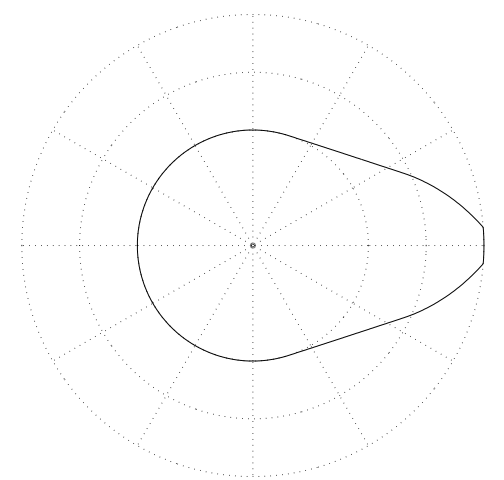

Figure 3.1: Cam shape for $\alpha=1.5$. 


\section{Hanging Chain}

Find the chain (of uniform density) of length $L$ suspended between two points with minimal potential energy.

\section{Implementation}

This classical problem (see Cesari [10, pages 126-127]) was suggested by Hans Mittelmann. In this problem we need to determine a function $x(t)$, the shape of the chain, that minimizes the potential energy

$$
\int_{0}^{1} x\left(1+\left(x^{\prime}\right)^{2}\right)^{1 / 2} d t
$$

subject to the constraint on the length of the chain,

$$
\int_{0}^{1}\left(1+\left(x^{\prime}\right)^{2}\right)^{1 / 2} d t=L
$$

and the end conditions $x(0)=a$ and $x(1)=b$. Various formulations of this problem are possible. We use a formulation in terms of the control $x_{1}^{\prime}=u$, and the partial potential energy function

$$
x_{2}(t)=\int_{0}^{t} x_{1}(s)\left(1+u(s)^{2}\right)^{1 / 2} d s .
$$

This definition leads to an end-point formulation of minimizing the total potential energy $x_{2}(1)$ subject to the differential equations

$$
x_{1}^{\prime}=u, \quad x_{2}^{\prime}=x_{1}\left(1+u^{2}\right)^{1 / 2}, \quad x_{3}^{\prime}=\left(1+u^{2}\right)^{1 / 2} .
$$

Discretization is done using a uniform time step and the trapezoidal rule for the integration of the system over $n_{h}$ intervals. Data for this problem appears in Table 4.1.

Table 4.1: Hanging chain problem data

\begin{tabular}{|l|c|}
\hline Variables & $4 n_{h}-2$ \\
Constraints & $3 n_{h}$ \\
Bounds & 0 \\
Linear equality constraints & $n_{h}$ \\
Linear inequality constraints & 0 \\
Nonlinear equality constraints & $2 n_{h}$ \\
Nonlinear inequality constraints & 0 \\
Nonzeros in $\nabla^{2} f(x)$ & 0 \\
Nonzeros in $c^{\prime}(x)$ & $14 n_{h}-7$ \\
\hline
\end{tabular}

\section{Performance}

Results for the AMPL implementation are summarized in Table 4.2 with $a=1, b=3$, and $L=4$. The starting point is the quadratic

$$
x_{1}(t)=(2|b-a|) t\left(t-2 t_{m}\right)+a,
$$


where $t_{m}=0.25$ if $b>a$ and $t_{m}=0.75$ otherwise, evaluated at the mesh points. This choice is convex and satisfies the boundary data. The control function $u$ is set to $x_{1}^{\prime}$, while $x_{2}(t)=x_{1}(t) u(t)$ and $x_{3}(t)=u(t)$. The optimal chain is shown in Figure 4.1.

Table 4.2: Performance on hanging chain problem

\begin{tabular}{|c|c|c|c|}
\hline Solver & $n_{h}=200$ & $n_{h}=400$ & $n_{h}=800$ \\
\hline \hline FILTER & $20.22 \mathrm{~s}$ & $35.35 \mathrm{~s}$ & $124.83 \mathrm{~s}$ \\
$f$ & $5.06891 \mathrm{e}+00$ & $5.06862 \mathrm{e}+00$ & $5.06852 \mathrm{e}+00$ \\
$c$ violation & $6.27 \mathrm{e}-12$ & $2.31 \mathrm{e}-10$ & $9.24 \mathrm{e}-10$ \\
optimality & $1.07 \mathrm{e}-08$ & $3.03 \mathrm{e}-08$ & $4.61 \mathrm{e}-08$ \\
\hline KNITRO & $0.13 \mathrm{~s}$ & $0.29 \mathrm{~s}$ & $0.59 \mathrm{~s}$ \\
$f$ & $5.06891 \mathrm{e}+00$ & $5.06862 \mathrm{e}+00$ & $5.06852 \mathrm{e}+00$ \\
$c$ violation & $8.42 \mathrm{e}-10$ & $5.59 \mathrm{e}-10$ & $3.34 \mathrm{e}-10$ \\
optimality & $6.58 \mathrm{e}-10$ & $4.16 \mathrm{e}-10$ & $2.15 \mathrm{e}-10$ \\
\hline LOQO & $0.37 \mathrm{~s}$ & $0.79 \mathrm{~s}$ & $1.75 \mathrm{~s}$ \\
$f$ & $5.06891 \mathrm{e}+00$ & $5.06862 \mathrm{e}+00$ & $5.06852 \mathrm{e}+00$ \\
$c$ violation & $4.22 \mathrm{e}-09$ & $5.40 \mathrm{e}-09$ & $8.47 \mathrm{e}-08$ \\
optimality & $3.43 \mathrm{e}-10$ & $4.75 \mathrm{e}-10$ & $1.52 \mathrm{e}-09$ \\
\hline MINOS & $8.02 \mathrm{~s}$ & $33.19 \mathrm{~s}$ & $150.54 \mathrm{~s}$ \\
$f$ & $5.06891 \mathrm{e}+00$ & $5.06862 \mathrm{e}+00$ & $5.06852 \mathrm{e}+00$ \\
$c$ violation & $2.51 \mathrm{e}-12$ & $1.79 \mathrm{e}-10$ & $1.16 \mathrm{e}-09$ \\
optimality & $3.54 \mathrm{e}-07$ & $8.32 \mathrm{e}-07$ & $1.59 \mathrm{e}-07$ \\
\hline SNOPT & $6.31 \mathrm{~s}$ & $42.71 \mathrm{~s}$ & $125.98 \mathrm{~s}$ \\
$f$ & $5.06891 \mathrm{e}+00$ & $5.06862 \mathrm{e}+00$ & $5.06852 \mathrm{e}+00$ \\
$c$ violation & $4.86 \mathrm{e}-09$ & $7.67 \mathrm{e}-10$ & $1.14 \mathrm{e}-09$ \\
optimality & $5.24 \mathrm{e}-07$ & $1.55 \mathrm{e}-07$ & $1.57 \mathrm{e}-07$ \\
\hline
\end{tabular}

$\dagger$ Errors. $\ddagger$ Timed out. $\uparrow$ Failed benchmark tests.

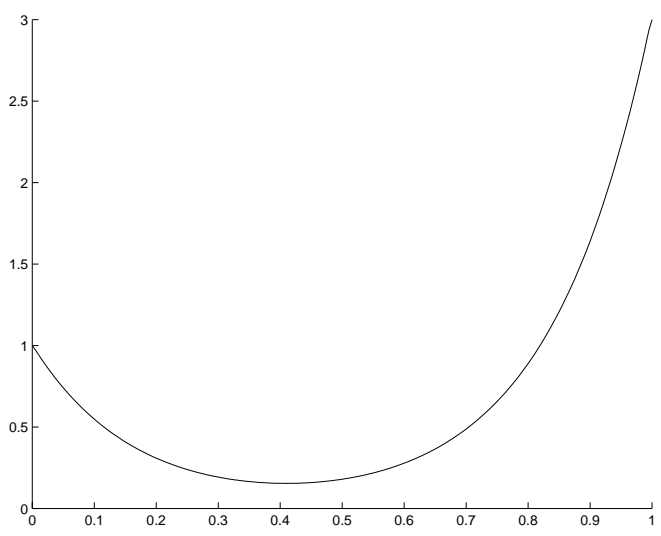

Figure 4.1: Hanging chain of length $L=4$ 


\section{Isometrization of $\alpha$-pinene}

Determine the reaction coefficients in the thermal isometrization of $\alpha$-pinene. The linear kinetic model [5] is defined in terms of reaction coefficients $\theta_{i} \geq 0$ via

$$
\begin{aligned}
& y_{1}^{\prime}=-\left(\theta_{1}+\theta_{2}\right) y_{1} \\
& y_{2}^{\prime}=\theta_{1} y_{1} \\
& y_{3}^{\prime}=\theta_{2} y_{1}-\left(\theta_{3}+\theta_{4}\right) y_{3}+\theta_{5} y_{5} \\
& y_{4}^{\prime}=\theta_{3} y_{3} \\
& y_{5}^{\prime}=\theta_{4} y_{3}-\theta_{5} y_{5},
\end{aligned}
$$

Initial conditions for (5.1) are known. The problem is to minimize

$$
\sum_{j=1}^{8}\left\|y\left(\tau_{j} ; \theta\right)-z_{j}\right\|^{2},
$$

where $z_{j}$ are concentration measurements for $y$ at time points $\tau_{1}, \ldots, \tau_{8}$.

\section{Formulation}

Our formulation of the $\alpha$-pinene problem as an optimization problem follows [31, 3]. We use a $k$-stage collocation method, a uniform partition with $n_{h}$ subintervals of $\left[0, \tau_{8}\right]$, and the standard [2, pages 247-249] basis representation,

$$
p_{\pi}(t)=v_{i}+\sum_{j=1}^{k} \frac{\left(t-t_{i}\right)^{j}}{j ! h^{j-1}} w_{i j}, \quad t \in\left[t_{i}, t_{i+1}\right],
$$

for the components of the solution $y$ of (5.1). The constraints in the optimization problem are the initial conditions in (5.1), the continuity conditions, and the collocation equations. The continuity equations at each interior grid point are a set of $5\left(n_{h}-1\right)$ linear equations. The collocation equations are a set of $5 k n_{h}$ nonlinear equations obtained by requiring that the collocation approximation satisfy (5.1) at the collocation points. Data for this problem appears in Table 5.1. The number of nonzeros in the Hessian of the objective function is an upper bound. Fewer nonzeros are present if the times at which the measurements were taken coincide with the grid points.

Table 5.1: Isometrization of $\alpha$-pinene data

\begin{tabular}{|l|c|}
\hline Variables & $5(k+1) h_{h}$ \\
Constraints & $5(k+1) n_{h}-5$ \\
Bounds & 5 \\
Linear equality constraints & $5 n_{h}-5$ \\
Linear inequality constraints & 0 \\
Nonlinear equality constraints & $5 k n_{h}$ \\
Nonlinear inequality constraints & 0 \\
Nonzeros in $\nabla^{2} f(x)$ & $40(k+1)^{2}$ \\
Nonzeros in $c^{\prime}(x)$ & $k(10 k+23) n_{h}+10 n_{h}-13 k-15$ \\
\hline
\end{tabular}




\section{Performance}

We provide results for the AMPL formulation with $k=3$ in Table 5.2. The initial values for the $\theta$ parameters are $\theta_{i}=0.0$. The initial basis parameters are chosen so that the collocation approximation is piecewise constant and interpolates the data. The solution and data are shown in Figure 5.1.

Table 5.2: Performance on isometrization problem

\begin{tabular}{|c|c|c|c|}
\hline Solver & $n_{h}=100$ & $n_{h}=200$ & $n_{h}=400$ \\
\hline \hline FILTER & $27.76 \mathrm{~s}$ & $214.03 \mathrm{~s}$ & $1296.29 \mathrm{~s}$ \\
$f$ & $1.98721 \mathrm{e}+01$ & $1.98721 \mathrm{e}+01$ & $1.98721 \mathrm{e}+01$ \\
$c$ violation & $2.21 \mathrm{e}-14$ & $3.58 \mathrm{e}-12$ & $3.91 \mathrm{e}-14$ \\
optimality & $9.83 \mathrm{e}-10$ & $2.28 \mathrm{e}-09$ & $2.00 \mathrm{e}-09$ \\
\hline KNITRO & $0.47 \mathrm{~s}$ & $1 \mathrm{~s}$ & $2.42 \mathrm{~s}$ \\
$f$ & $1.98721 \mathrm{e}+01$ & $1.98721 \mathrm{e}+01$ & $1.98721 \mathrm{e}+01$ \\
$c$ violation & $2.18 \mathrm{e}-11$ & $1.94 \mathrm{e}-11$ & $6.78 \mathrm{e}-12$ \\
optimality & $9.58 \mathrm{e}-08$ & $1.52 \mathrm{e}-08$ & $1.42 \mathrm{e}-08$ \\
\hline LOQO & $1 \mathrm{~s}$ & $2.09 \mathrm{~s}$ & $4.83 \mathrm{~s}$ \\
$f$ & $1.98721 \mathrm{e}+01$ & $1.98721 \mathrm{e}+01$ & $1.98721 \mathrm{e}+01$ \\
$c$ violation & $1.06 \mathrm{e}-11$ & $8.80 \mathrm{e}-12$ & $2.35 \mathrm{e}-12$ \\
optimality & $2.79 \mathrm{e}-08$ & $1.47 \mathrm{e}-09$ & $2.86 \mathrm{e}-09$ \\
\hline MINOS & $2.19 \mathrm{~s}$ & $20.62 \mathrm{~s}$ & $53.47 \mathrm{~s}$ \\
$f$ & $1.98721 \mathrm{e}+01$ & $4.38234 \mathrm{e}+17 \dagger$ & $1.98721 \mathrm{e}+01$ \\
$c$ violation & $3.67 \mathrm{e}-10$ & $1.00 \mathrm{e}+00 \dagger$ & $7.71 \mathrm{e}-10$ \\
optimality & $2.15 \mathrm{e}-08$ & $1.00 \mathrm{e}+00 \dagger$ & $5.45 \mathrm{e}-07$ \\
\hline SNOPT & $8.47 \mathrm{~s}$ & $31.61 \mathrm{~s}$ & $96.07 \mathrm{~s}$ \\
$f$ & $1.98721 \mathrm{e}+01$ & $1.98721 \mathrm{e}+01 \dagger$ & $1.98721 \mathrm{e}+01$ \\
$c$ violation & $1.07 \mathrm{e}-09$ & $4.29 \mathrm{e}-10 \dagger$ & $1.29 \mathrm{e}-09$ \\
optimality & $4.28 \mathrm{e}-07$ & $7.18 \mathrm{e}-07 \dagger$ & $2.03 \mathrm{e}-09$ \\
\hline
\end{tabular}

$\dagger$ Errors. + Timed out. $\uparrow$ Failed benchmark tests.

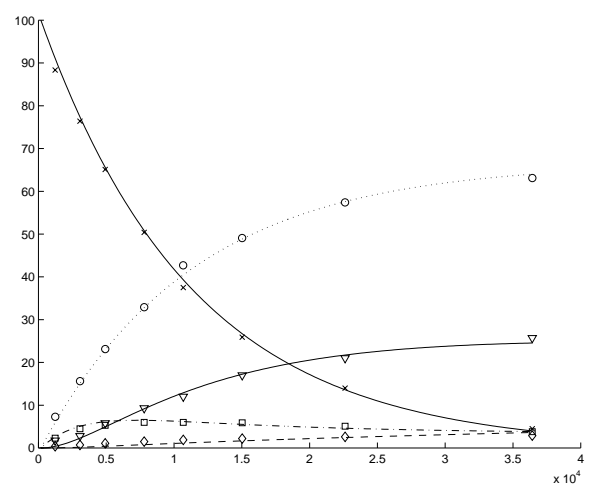

Figure 5.1: Solution and data for the $\alpha$-pinene problem 


\section{Marine Population Dynamics}

Given estimates of the abundance of the population of a marine species at each stage (for example, nauplius, juvenile, adult) as a function of time, determine stage specific growth and mortality rates. The model for the population dynamics of the $n_{s}$-stage population is

$$
y_{j}^{\prime}=g_{j-1} y_{j-1}-\left(m_{j}+g_{j}\right) y_{j}, \quad 1 \leq j \leq n_{s},
$$

where $m_{i}$ and $g_{i}$ are the unknown mortality and growth rates at stage $i$ with $g_{0}=g_{n_{s}}=0$. This model assumes that the species eventually dies or grows into the next stage, with the implicit assumption that the species cannot skip a stage. Initial conditions for the differential equations are unknown, since the stage abundance measurements at the initial time might also be contaminated with experimental error. We minimize the error between computed and observed data,

$$
\sum_{j=1}^{n_{m}}\left\|y\left(\tau_{j} ; m, g\right)-z_{j}\right\|^{2}
$$

where $z_{j}$ are the stage abundance measurements. This problem is based on the work of Rothschild, Sharov, Kearsley, and Bondarenko [26].

\section{Formulation}

Our formulation of the marine population dynamics uses a $k$-stage collocation method, a uniform partition with $n_{h}$ subintervals of $\left[0, \tau_{n_{m}}\right]$, and the standard [2, pages 247-249] basis representation,

$$
p_{\pi}(t)=v_{i}+\sum_{j=1}^{k} \frac{\left(t-t_{i}\right)^{j}}{j ! h^{j-1}} w_{i j}, \quad t \in\left[t_{i}, t_{i+1}\right],
$$

for the components of the solution $y$ of (6.1). The constraints in the optimization problem are the continuity conditions and the collocation equations. The continuity equations are a set of $n_{s}\left(n_{h}-1\right)$ linear equations. The collocation equations are a set of $k n_{s} n_{h}$ nonlinear equations obtained by requiring that the collocation approximation satisfy (6.1) at the collocation points.

Table 6.1: Marine population dynamics problem data

\begin{tabular}{|l|c|}
\hline Variables & $(k+1) n_{s} n_{h}+2 n_{s}-1$ \\
Constraints & $(k+1) n_{s} n_{h}-n_{s}$ \\
Bounds & $2 n_{s}-1$ \\
Linear equality constraints & $n_{s}\left(n_{h}-1\right)$ \\
Linear inequality constraints & 0 \\
Nonlinear equality constraints & $k n_{s} n_{h}$ \\
Nonlinear inequality constraints & 0 \\
Nonzeros in $\nabla^{2} f(x)$ & $(k+1)^{2} n_{s} n_{m}$ \\
Nonzeros in $c^{\prime}(x)$ & $\left(k^{2}+3 k+1\right)\left(2 n_{s}-1\right) n_{h}+n_{h}-(k+2) n_{s}$ \\
\hline
\end{tabular}


The parameters in the problem are the $n_{s} n_{h}$ initial conditions, the $n_{s}$ mortality rates, the $n_{s}-1$ growth rates, and the $k n_{s} n_{h}$ basis parameters in the representation of the collocation approximation. Data for this problem appears in Table 6.1. The number of nonzeros in the Hessian of the objective function is an upper bound. Fewer nonzeros are present if the times at which the measurements were taken coincide with the grid points.

We do not impose any initial conditions on the differential equations, since initial measurements are usually contaminated with experimental error. Introducing these extra degrees of freedom into the problem formulation should allow solvers to find a better fit to the data. A significant difference between this problem and other parameter estimation problems is that the population dynamics data usually contains large observation errors.

\section{Performance}

We provide results for the AMPL formulation with $k=1$ in Table 6.2. We use a simulated dataset with $n_{s}=8$ stages. The initial basis parameters are chosen so that the collocation approximation is piecewise constant and interpolates the data. For this problem we are using a single collocation point $(k=1)$, since in this case the number of parameters grows quickly with the number of stages. The quality of the solution does not seem to be affected, at least as measured by the population curves and the mortality and growth parameters.

Table 6.2: Performance on marine population dynamics problem

\begin{tabular}{|c|c|c|c|}
\hline Solver & $n_{h}=100$ & $n_{h}=200$ & $n_{h}=400$ \\
\hline \hline FILTER & $26.62 \mathrm{~s}$ & $155.97 \mathrm{~s}$ & $856.31 \mathrm{~s}$ \\
$f$ & $1.97462 \mathrm{e}+07$ & $1.97464 \mathrm{e}+07$ & $1.97465 \mathrm{e}+07$ \\
$c$ violation & $8.14 \mathrm{e}-12$ & $1.61 \mathrm{e}-11$ & $2.03 \mathrm{e}-11$ \\
optimality & $2.75 \mathrm{e}-08$ & $1.56 \mathrm{e}-08$ & $3.75 \mathrm{e}-08$ \\
\hline KNITRO & $1.02 \mathrm{~s}$ & $1.95 \mathrm{~s}$ & $4.65 \mathrm{~s}$ \\
$f$ & $1.97462 \mathrm{e}+07$ & $1.97464 \mathrm{e}+07$ & $1.97465 \mathrm{e}+07$ \\
$c$ violation & $4.13 \mathrm{e}-10$ & $7.63 \mathrm{e}-10$ & $9.80 \mathrm{e}-08$ \\
optimality & $1.09 \mathrm{e}-07$ & $5.80 \mathrm{e}-08$ & $3.91 \mathrm{e}-08$ \\
\hline LOQO & $0.89 \mathrm{~s}$ & $2.07 \mathrm{~s}$ & $5.4 \mathrm{~s}$ \\
$f$ & $1.97462 \mathrm{e}+07$ & $1.97464 \mathrm{e}+07$ & $1.97465 \mathrm{e}+07$ \\
$c$ violation & $4.24 \mathrm{e}-10$ & $1.21 \mathrm{e}-09$ & $7.37 \mathrm{e}-09$ \\
optimality & $1.02 \mathrm{e}-07$ & $1.06 \mathrm{e}-07$ & $3.09 \mathrm{e}-07$ \\
\hline MINOS & $1.12 \mathrm{~s}$ & $1.29 \mathrm{~s}$ & $2.98 \mathrm{~s}$ \\
$f$ & $1.97462 \mathrm{e}+07$ & $4.45228 \mathrm{e}+141 \dagger$ & $2.66644 \mathrm{e}+72 \dagger$ \\
$c$ violation & $3.21 \mathrm{e}-10$ & $1.00 \mathrm{e}+00 \dagger$ & $1.00 \mathrm{e}+00 \dagger$ \\
optimality & $8.32 \mathrm{e}-08$ & $1.00 \mathrm{e}+00 \dagger$ & $1.00 \mathrm{e}+00 \dagger$ \\
\hline SNOPT & $5.66 \mathrm{~s}$ & $14.35 \mathrm{~s}$ & $57.16 \mathrm{~s}$ \\
$f$ & $1.97462 \mathrm{e}+07 \dagger$ & $1.97464 \mathrm{e}+07$ & $1.97465 \mathrm{e}+07$ \\
$c$ violation & $7.27 \mathrm{e}-12 \dagger$ & $8.18 \mathrm{e}-12$ & $9.09 \mathrm{e}-12$ \\
optimality & $3.74 \mathrm{e}-07 \dagger$ & $3.09 \mathrm{e}-07$ & $3.20 \mathrm{e}-07$ \\
\hline
\end{tabular}

$\dagger$ Errors. $†$ Timed out. $\uparrow$ Failed benchmark tests.

The graph on the left of Figure 6.1 shows the populations for stages 1,2,5, and 6 , while the graph on the right shows the populations for stages $3,4,7$, and 8 . In both cases, the fit between the model and the data is not always tight. Figure 6.2 plots the mortality and 
growth parameters for the eight stages. Mortality parameters are marked $*$, while growth parameters are marked $\circ$. The mortality parameters for stages 5 and 6 are not zero, but they are on the order of $10^{-3}$ and $10^{-9}$, respectively.
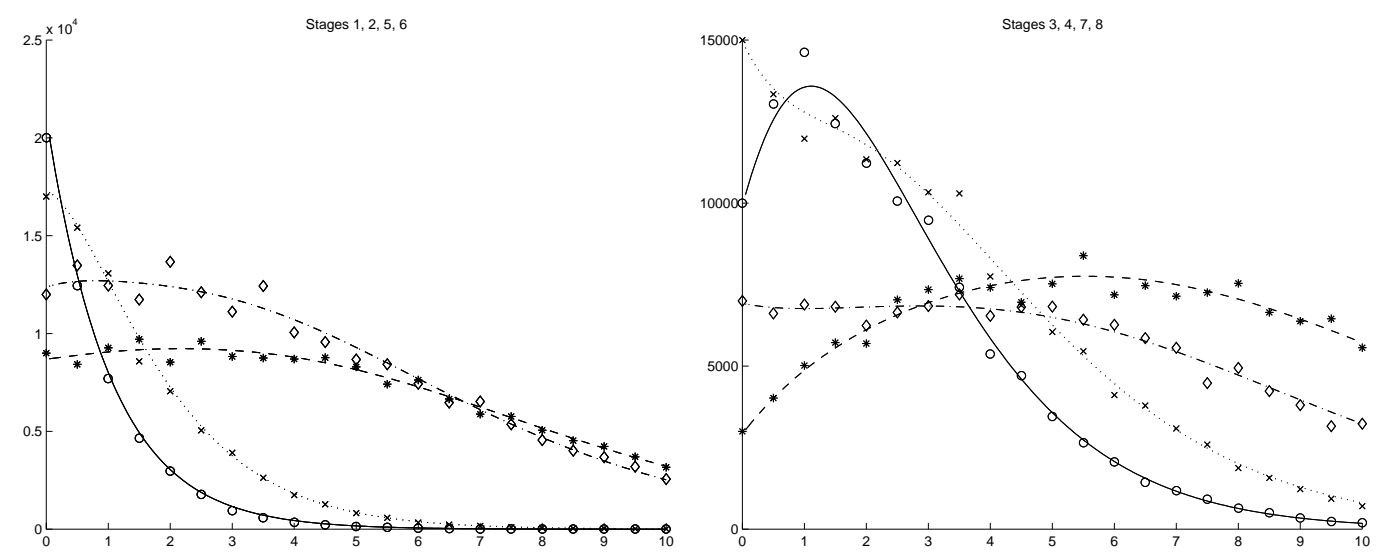

Figure 6.1: Marine populations for stages 1,2,5,6 (left) and 3, 4,7,8 (right)

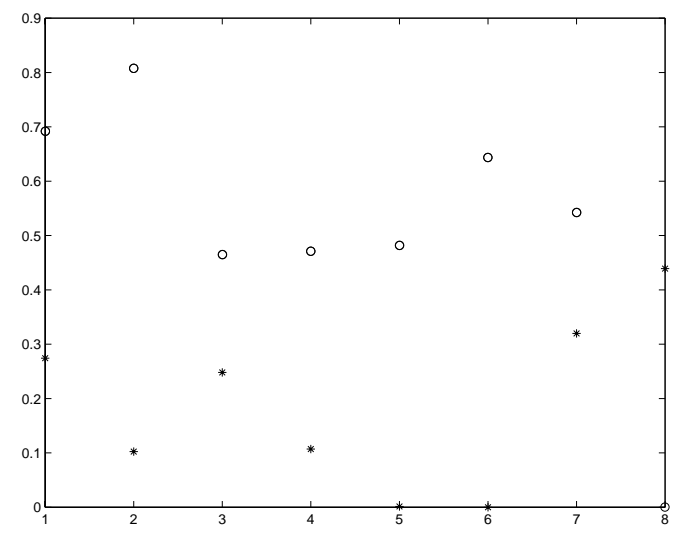

Figure 6.2: Mortality $(*)$ and growth $(\circ)$ parameters for the marine populations stages 


\section{Flow in a Channel}

Analyze the flow of a fluid during injection into a long vertical channel, assuming that the flow is modeled by the boundary value problem

$$
\begin{aligned}
& u^{\prime \prime \prime \prime}=R\left(u^{\prime} u^{\prime \prime}-u u^{\prime \prime \prime}\right), \quad 0 \leq t \leq 1, \\
& u(0)=0, \quad u(1)=1, \quad u^{\prime}(0)=u^{\prime}(1)=0,
\end{aligned}
$$

where $u$ is the potential function, $u^{\prime}$ is the tangential velocity of the fluid, and $R$ is the Reynolds number.

\section{Formulation}

We use a $k$-stage collocation method to formulate this problem as an optimization problem with a constant merit function and equality constraints representing the solution of (7.1). We use a uniform partition with $n_{h}$ subintervals of [0,1], and the standard [2, pages 247-249] basis representation,

$$
p_{\pi}(t)=\sum_{j=1}^{m} \frac{\left(t-t_{i}\right)^{j-1}}{(j-1) !} v_{i j}+\sum_{j=1}^{k} \frac{\left(t-t_{i}\right)^{j+m-1}}{(j+m-1) ! h^{j-1}} w_{i j}, \quad t \in\left[t_{i}, t_{i+1}\right],
$$

for $u$. We require the continuity conditions that $p_{\pi} \in C^{m-1}$, where $m$ is the order of the differential equation.

The constraints in the optimization problem are the initial conditions in (7.1), the continuity conditions, and the collocation equations. There are $m=4$ initial conditions. The continuity equations are a set of $m\left(n_{h}-1\right)$ linear equations. The collocation equations are a set of $k n_{h}$ nonlinear equations obtained by requiring that $u$ satisfy (7.1) at the collocation points $\xi_{i j}=t_{i}+h \rho_{j}$ for $i=1, \ldots, n_{h}$ and $j=1, \ldots, k$. The collocation points $\rho_{j}$ are the roots of the $k$ th degree Legendre polynomial. The parameters in the optimization problem are the $(m+k) n_{h}$ parameters $v_{i j}$ and $w_{i j}$ in the representation of $u$. Data for this problem appears in Table 7.1.

Table 7.1: Flow in a channel problem data

\begin{tabular}{|l|c|}
\hline Variables & $(k+4) n_{h}-2$ \\
Constraints & $(k+4) n_{h}-2$ \\
Bounds & 0 \\
Linear equality constraints & $4 n_{h}-2$ \\
Linear inequality constraints & 0 \\
Nonlinear equality constraints & $k n_{h}$ \\
Nonlinear inequality constraints & 0 \\
Nonzeros in $\nabla^{2} f(x)$ & 0 \\
Nonzeros in $c^{\prime}(x)$ & $\left(k^{2}+8 k+14\right) n_{h}-4 k-10$ \\
\hline
\end{tabular}




\section{Performance}

Results for the AMPL implementation with $k=4$ and $R=10$ are summarized in Table 7.2. The starting point is the function $t^{2}(3-2 t)$ evaluated at the mesh points. Solutions for several $R$ are shown in Figure 7.1. This problem is easy to solve for small Reynolds numbers but becomes increasingly difficult to solve as $R$ increases.

Table 7.2: Performance on flow in channel problem

\begin{tabular}{|c|c|c|c|}
\hline Solver & $n_{h}=200$ & $n_{h}=400$ & $n_{h}=800$ \\
\hline \hline FILTER & $9.61 \mathrm{~s}$ & $56.77 \mathrm{~s}$ & $343.61 \mathrm{~s}$ \\
$f$ & $1.00000 \mathrm{e}+00$ & $1.00000 \mathrm{e}+00$ & $1.00000 \mathrm{e}+00$ \\
$c$ violation & $1.29 \mathrm{e}-11$ & $3.54 \mathrm{e}-14$ & $2.64 \mathrm{e}-11$ \\
optimality & $0.00 \mathrm{e}+00$ & $0.00 \mathrm{e}+00$ & $0.00 \mathrm{e}+00$ \\
\hline KNITRO & $0.63 \mathrm{~s}$ & $1.31 \mathrm{~s}$ & $2.71 \mathrm{~s}$ \\
$f$ & $1.00000 \mathrm{e}+00$ & $1.00000 \mathrm{e}+00$ & $1.00000 \mathrm{e}+00$ \\
$c$ violation & $2.27 \mathrm{e}-12$ & $2.26 \mathrm{e}-12$ & $2.35 \mathrm{e}-12$ \\
optimality & $0.00 \mathrm{e}+00$ & $0.00 \mathrm{e}+00$ & $0.00 \mathrm{e}+00$ \\
\hline LOQO & $1.29 \mathrm{~s}$ & $3.26 \mathrm{~s}$ & $8.81 \mathrm{~s}$ \\
$f$ & $1.00000 \mathrm{e}+00$ & $1.00000 \mathrm{e}+00$ & $1.00000 \mathrm{e}+00$ \\
$c$ violation & $8.80 \mathrm{e}-10$ & $4.85 \mathrm{e}-09$ & $4.37 \mathrm{e}-09$ \\
optimality & $0.00 \mathrm{e}+00$ & $0.00 \mathrm{e}+00$ & $0.00 \mathrm{e}+00$ \\
\hline MINOS & $1.16 \mathrm{~s}$ & $4.04 \mathrm{~s}$ & $13.08 \mathrm{~s}$ \\
$f$ & $1.00000 \mathrm{e}+00$ & $1.00000 \mathrm{e}+00$ & $1.00000 \mathrm{e}+00$ \\
$c$ violation & $2.01 \mathrm{e}-13$ & $3.47 \mathrm{e}-13$ & $4.70 \mathrm{e}-08$ \\
optimality & $0.00 \mathrm{e}+00$ & $0.00 \mathrm{e}+00$ & $0.00 \mathrm{e}+00$ \\
\hline SNOPT & $5.3 \mathrm{~s}$ & $17.46 \mathrm{~s}$ & $57.77 \mathrm{~s}$ \\
$f$ & $1.00000 \mathrm{e}+00$ & $1.00000 \mathrm{e}+00 \dagger$ & $1.00000 \mathrm{e}+00 \dagger$ \\
$c$ violation & $4.82 \mathrm{e}-13$ & $3.82 \mathrm{e}-07 \dagger$ & $4.99 \mathrm{e}-07 \dagger$ \\
optimality & $0.00 \mathrm{e}+00$ & $0.00 \mathrm{e}+00 \dagger$ & $0.00 \mathrm{e}+00 \dagger$ \\
\hline
\end{tabular}

$\dagger$ Errors. + Timed out. $\uparrow$ Failed benchmark tests.

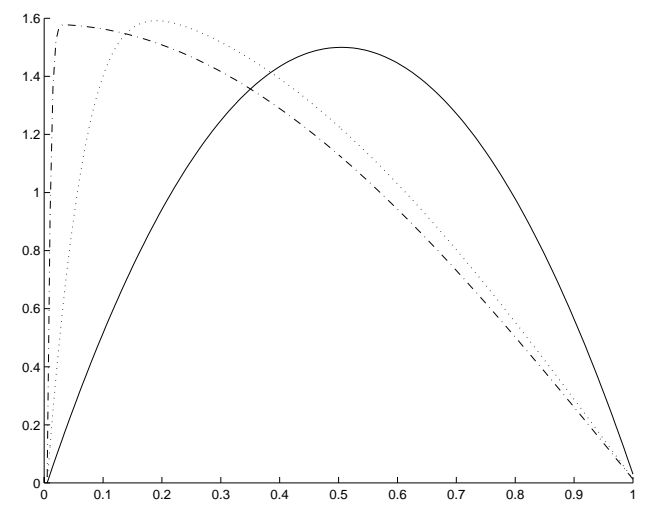

Figure 7.1: Tangential velocity $u^{\prime}$ for Reynolds numbers $R=0,10^{2}, 10^{4}$ 


\section{Robot Arm}

Minimize the time taken for a robot arm to travel between two points.

\section{Formulation}

This problem originated in the thesis of Monika Mössner-Beigel (Heidelberg University). In her formulation the arm of the robot is a rigid bar of length $L$ that protrudes a distance $\rho$ from the origin to the gripping end and sticks out a distance $L-\rho$ in the opposite direction. If the pivot point of the arm is the origin of a spherical coordinate system, then the problem can be phrased in terms of the length $\rho$ of the arm from the pivot, the horizontal and vertical angles $(\theta, \phi)$ from the horizontal plane, the controls $\left(u_{\rho}, u_{\theta}, u_{\phi}\right)$, and the final time $t_{f}$. Bounds on the length and angles are

$$
\rho(t) \in[0, L], \quad|\theta(t)| \leq \pi, \quad 0 \leq \phi(t) \leq \pi,
$$

and for the controls,

$$
\left|u_{\rho}\right| \leq 1, \quad\left|u_{\theta}\right| \leq 1, \quad\left|u_{\phi}\right| \leq 1 .
$$

The equations of motion for the robot arm are

$$
L \rho^{\prime \prime}=u_{\rho}, \quad I_{\theta} \theta^{\prime \prime}=u_{\theta}, \quad I_{\phi} \phi^{\prime \prime}=u_{\phi},
$$

where $I$ is the moment of inertia, defined by

$$
I_{\theta}=\frac{\left((L-\rho)^{3}+\rho^{3}\right)}{3} \sin (\phi)^{2}, \quad I_{\phi}=\frac{\left((L-\rho)^{3}+\rho^{3}\right)}{3} .
$$

The boundary conditions are

$$
\begin{gathered}
\rho(0)=\rho\left(t_{f}\right)=4.5, \quad \theta(0)=0, \theta\left(t_{f}\right)=\frac{2 \pi}{3}, \quad \phi(0)=\phi\left(t_{f}\right)=\frac{\pi}{4}, \\
\rho^{\prime}(0)=\theta^{\prime}(0)=\phi^{\prime}(0)=\rho^{\prime}\left(t_{f}\right)=\theta^{\prime}\left(t_{f}\right)=\phi^{\prime}\left(t_{f}\right)=0 .
\end{gathered}
$$

This model ignores the fact that the spherical coordinate reference frame is a noninertial frame and should have terms for Coriolis and centrifugal forces.

\section{Implementation}

In the implementation of Vanderbei [32] the controls $u$ are eliminated by substitution, and thus the equality constraints in (8.1) become the inequalities

$$
\left|L \rho^{\prime \prime}\right| \leq 1, \quad\left|I_{\theta} \theta^{\prime \prime}\right| \leq 1, \quad\left|I_{\phi} \phi^{\prime \prime}\right| \leq 1
$$

In this implementation (8.1) is expressed in terms of a first-order system with the additional variables $\rho^{\prime}, \theta^{\prime}$, and $\phi^{\prime}$. Discretization is done with a uniform time step and the trapezoidal rule over $n_{h}$ intervals. Data for this problem is shown in Table 8.1. 
Table 8.1: Robot arm problem data

\begin{tabular}{|l|c|}
\hline Variables & $9\left(n_{h}+1\right)-11$ \\
Constraints & $6 n_{h}$ \\
Bounds & $6 n_{h}$ \\
Linear equality constraints & 0 \\
Linear inequality constraints & 0 \\
Nonlinear equality constraints & $6 n_{h}$ \\
Nonlinear inequality constraints & 0 \\
Nonzeros in $\nabla^{2} f(x)$ & 0 \\
Nonzeros in $c^{\prime}(x)$ & $36 n_{h}-26$ \\
\hline
\end{tabular}

\section{Performance}

Results for the AMPL implementation appear in Table 8.2. All solvers were given the same initial values. The initial values for $\rho$ and $\phi$ were set to the functions $\rho \equiv 4.5$ and $\phi \equiv \pi / 4$ evaluated at the grid points. Similarly, initial values for $\theta$ were set to the discrete version of the parabola

$$
\theta(t)=\frac{2 \pi}{3}\left(\frac{t}{t_{f}}\right)^{2},
$$

which matches three of the boundary conditions. The initial values for all the controls were set to zero, and $t_{f}=1$ initially.

Table 8.2: Performance on robotic arm problem

\begin{tabular}{|c|c|c|c|}
\hline Solver & $n_{h}=200$ & $n_{h}=400$ & $n_{h}=800$ \\
\hline \hline FILTER & $6.09 \mathrm{~s}$ & $34.32 \mathrm{~s}$ & $182.28 \mathrm{~s}$ \\
$f$ & $9.14138 \mathrm{e}+00$ & $9.14101 \mathrm{e}+00$ & $9.14093 \mathrm{e}+00$ \\
$c$ violation & $1.43 \mathrm{e}-09$ & $1.51 \mathrm{e}-10$ & $4.02 \mathrm{e}-15$ \\
optimality & $2.12 \mathrm{e}-14$ & $4.48 \mathrm{e}-14$ & $3.10 \mathrm{e}-14$ \\
\hline KNITRO & $0.98 \mathrm{~s}$ & $2.58 \mathrm{~s}$ & $6.25 \mathrm{~s}$ \\
$f$ & $9.14138 \mathrm{e}+00$ & $9.14101 \mathrm{e}+00$ & $9.14093 \mathrm{e}+00$ \\
$c$ violation & $1.38 \mathrm{e}-11$ & $2.47 \mathrm{e}-13$ & $1.24 \mathrm{e}-13$ \\
optimality & $3.29 \mathrm{e}-14$ & $2.66 \mathrm{e}-14$ & $3.64 \mathrm{e}-14$ \\
\hline LOQO & $364.28 \mathrm{~s}$ & $207.62 \mathrm{~s}$ & $\ddagger$ \\
$f$ & $-1.98956 \mathrm{e}+03 \dagger$ & $-1.79892 \mathrm{e}+07 \dagger$ & $\dagger$ \\
$c$ violation & $1.00 \mathrm{e}+00 \dagger$ & $2.16 \mathrm{e}-07 \dagger$ & $\ddagger$ \\
optimality & $9.99 \mathrm{e}-01 \dagger$ & $1.00 \mathrm{e}+00 \dagger$ & $\ddagger$ \\
\hline MINOS & $6.38 \mathrm{~s}$ & $25.81 \mathrm{~s}$ & $107.21 \mathrm{~s}$ \\
$f$ & $9.14138 \mathrm{e}+00$ & $9.14101 \mathrm{e}+00$ & $9.14125 \mathrm{e}+00 \dagger$ \\
$c$ violation & $2.67 \mathrm{e}-12$ & $2.34 \mathrm{e}-12$ & $1.18 \mathrm{e}-11 \dagger$ \\
optimality & $5.32 \mathrm{e}-15$ & $3.55 \mathrm{e}-15$ & $2.63 \mathrm{e}-05 \dagger$ \\
\hline SNOPT & $2.86 \mathrm{~s}$ & $10.17 \mathrm{~s}$ & $102.32 \mathrm{~s}$ \\
$f$ & $9.14138 \mathrm{e}+00$ & $9.14101 \mathrm{e}+00$ & $9.14093 \mathrm{e}+00$ \\
$c$ violation & $4.44 \mathrm{e}-09$ & $4.76 \mathrm{e}-09$ & $2.86 \mathrm{e}-13$ \\
optimality & $6.21 \mathrm{e}-15$ & $1.34 \mathrm{e}-14$ & $6.57 \mathrm{e}-14$ \\
\hline
\end{tabular}

$\dagger$ Errors. $\ddagger$ Timed out. $\uparrow$ Failed benchmark tests. 
Figure 8.1 shows the variables $\rho, \theta, \phi$ for the robot arm as a function of time. We also show in Figure 8.2 the controls $u_{\rho}, u_{\theta}, u_{\phi}$ as a function of time. Note that the controls for the robot arm are bang-bang. Also note that the functions $\rho, \theta, \phi$ for the robot arm are continuously differentiable, but since the second derivatives are directly proportional to the controls, the second derivatives are piecewise continuous.
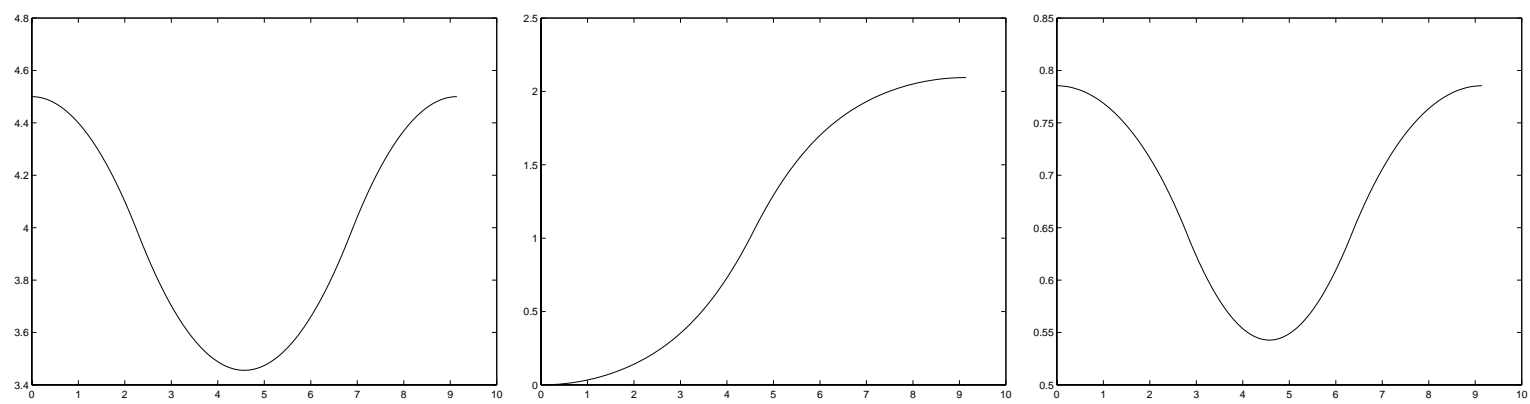

Figure 8.1: Variables $\rho, \theta, \phi$ for the robot arm as a function of time
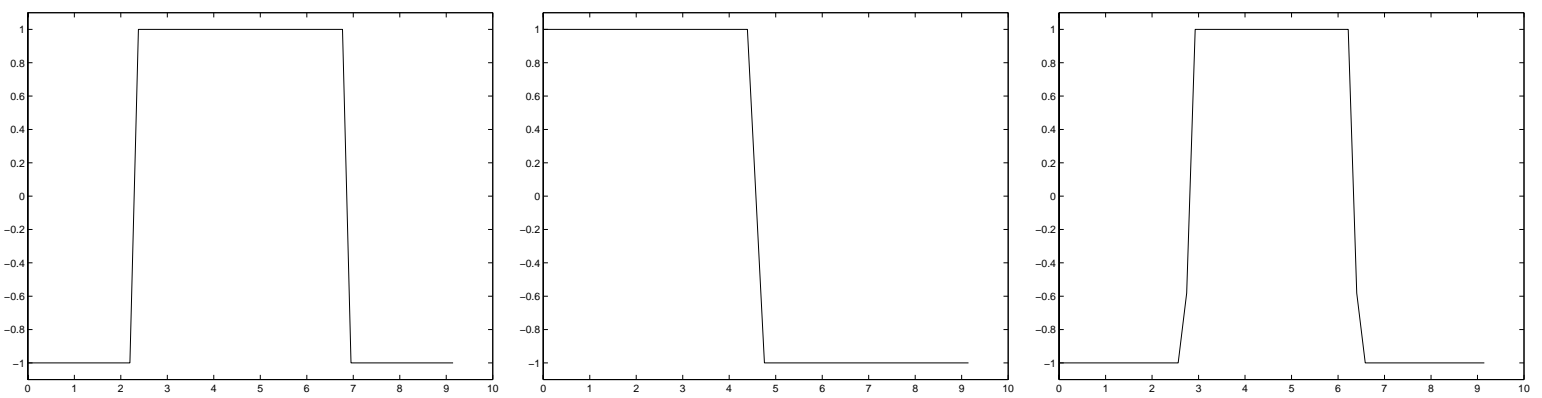

Figure 8.2: Control variables $u_{\rho}, u_{\theta}, u_{\phi}$ for the robot arm as a function of time 


\section{$9 \quad$ Particle Steering}

Minimize the time taken for a particle, acted upon by a thrust of constant magnitude, to achieve a given altitude and terminal velocity.

\section{Formulation}

The equations of motion are

$$
\ddot{y}_{1}=a \cos (u), \quad \ddot{y}_{2}=a \sin (u),
$$

where $\left(y_{1}, y_{2}\right)$ is the position of the particle, $u$ is the control angle with

$$
|u(t)| \leq \frac{\pi}{2}
$$

and $a$ is the constant magnitude of thrust. The particle is initially at rest so that

$$
y_{1}(0)=y_{2}(0)=\dot{y}_{1}(0)=\dot{y}_{2}(0)=0 .
$$

The problem is to minimize the travel time $t_{f}$ so that the particle achieves a given height $y_{2}\left(t_{f}\right)$ and terminal velocity $\left(\dot{y}_{1}\left(t_{f}\right), \dot{y}_{2}\left(t_{f}\right)\right)$.

This is a classical (see Bryson and Ho [6, pages 59-62]) problem in dynamic optimization. We use $a=100$ for the magnitude of thrust and the boundary conditions [4]

$$
y_{2}\left(t_{f}\right)=5, \quad \dot{y}_{1}\left(t_{f}\right)=45, \quad \dot{y}_{2}\left(t_{f}\right)=0 .
$$

Discretization is done using a uniform time step and the trapezoidal rule for the integration of the system over $n_{h}$ intervals. Data for this problem is shown in Table 9.1.

Table 9.1: Particle steering problem data

\begin{tabular}{|l|c|}
\hline Variables & $5 n_{h}-1$ \\
Constraints & $4 n_{h}$ \\
Bounds & $n_{h}+2$ \\
Linear equality constraints & 0 \\
Linear inequality constraints & 0 \\
Nonlinear equality constraints & $4 n_{h}$ \\
Nonlinear inequality constraints & 0 \\
Nonzeros in $\nabla^{2} f(x)$ & 0 \\
Nonzeros in $c^{\prime}(x)$ & $20 n_{h}-11$ \\
\hline
\end{tabular}

\section{Performance}

Results for the AMPL implementation are given in Table 9.2. The initial values for $y_{2}$ and $y_{3}=\dot{y}_{1}$ are chosen as the functions

$$
y_{1}(t)=5\left(\frac{t}{t_{f}}\right), \quad y_{3}(t)=45\left(\frac{t}{t_{f}}\right) .
$$


Table 9.2: Performance on particle steering problem

\begin{tabular}{|c|c|c|c|}
\hline Solver & $n_{h}=200$ & $n_{h}=400$ & $n_{h}=800$ \\
\hline \hline FILTER & $16.88 \mathrm{~s}$ & $73.36 \mathrm{~s}$ & $840.4 \mathrm{~s}$ \\
$f$ & $5.54577 \mathrm{e}-01$ & $5.54572 \mathrm{e}-01$ & $5.54571 \mathrm{e}-01$ \\
$c$ violation & $1.63 \mathrm{e}-09$ & $5.80 \mathrm{e}-11$ & $6.09 \mathrm{e}-10$ \\
optimality & $4.89 \mathrm{e}-08$ & $1.67 \mathrm{e}-08$ & $4.79 \mathrm{e}-08$ \\
\hline KNITRO & $0.32 \mathrm{~s}$ & $0.59 \mathrm{~s}$ & $1.55 \mathrm{~s}$ \\
$f$ & $5.54577 \mathrm{e}-01$ & $5.54572 \mathrm{e}-01$ & $5.54571 \mathrm{e}-01$ \\
$c$ violation & $4.32 \mathrm{e}-08$ & $1.74 \mathrm{e}-10$ & $1.27 \mathrm{e}-08$ \\
optimality & $1.50 \mathrm{e}-07$ & $7.68 \mathrm{e}-07$ & $3.20 \mathrm{e}-08$ \\
\hline LOQO & $1159.27 \mathrm{~s}$ & $\ddagger$ & $\ddagger$ \\
$f$ & $7.22390 \mathrm{e}-01 \dagger$ & $\ddagger$ & $\ddagger$ \\
$c$ violation & $1.24 \mathrm{e}-01 \dagger$ & $\ddagger$ & $\ddagger$ \\
optimality & $2.27 \mathrm{e}-03 \dagger$ & $\ddagger$ & $\ddagger$ \\
\hline MINOS & $4.02 \mathrm{~s}$ & $19.58 \mathrm{~s}$ & $13.54 \mathrm{~s}$ \\
$f$ & $5.54577 \mathrm{e}-01$ & $5.54572 \mathrm{e}-01$ & $0.00000 \mathrm{e}+00 \dagger$ \\
$c$ violation & $4.11 \mathrm{e}-08$ & $1.20 \mathrm{e}-08$ & $1.00 \mathrm{e}+00 \dagger$ \\
optimality & $5.58 \mathrm{e}-08$ & $1.10 \mathrm{e}-07$ & $5.40 \mathrm{e}-15 \dagger$ \\
\hline SNOPT & $10.02 \mathrm{~s}$ & $49.57 \mathrm{~s}$ & $245.19 \mathrm{~s}$ \\
$f$ & $5.54577 \mathrm{e}-01$ & $5.54572 \mathrm{e}-01$ & $5.54571 \mathrm{e}-01$ \\
$c$ violation & $5.98 \mathrm{e}-10$ & $6.68 \mathrm{e}-10$ & $5.71 \mathrm{e}-10$ \\
optimality & $7.12 \mathrm{e}-08$ & $1.15 \mathrm{e}-07$ & $1.29 \mathrm{e}-07$ \\
\hline
\end{tabular}

$\dagger$ Errors. $\ddagger$ Timed out. $\downarrow$ Failed benchmark tests.

Initial values for $y_{2}, y_{4}=\dot{y}_{2}$, and $u$ are set to zero. The initial value for the final time is $t_{f}=1$. Plots of the height $y_{2}$ and control $u$ as a function of the horizontal position $y_{1}$ are in Figure 9.1.
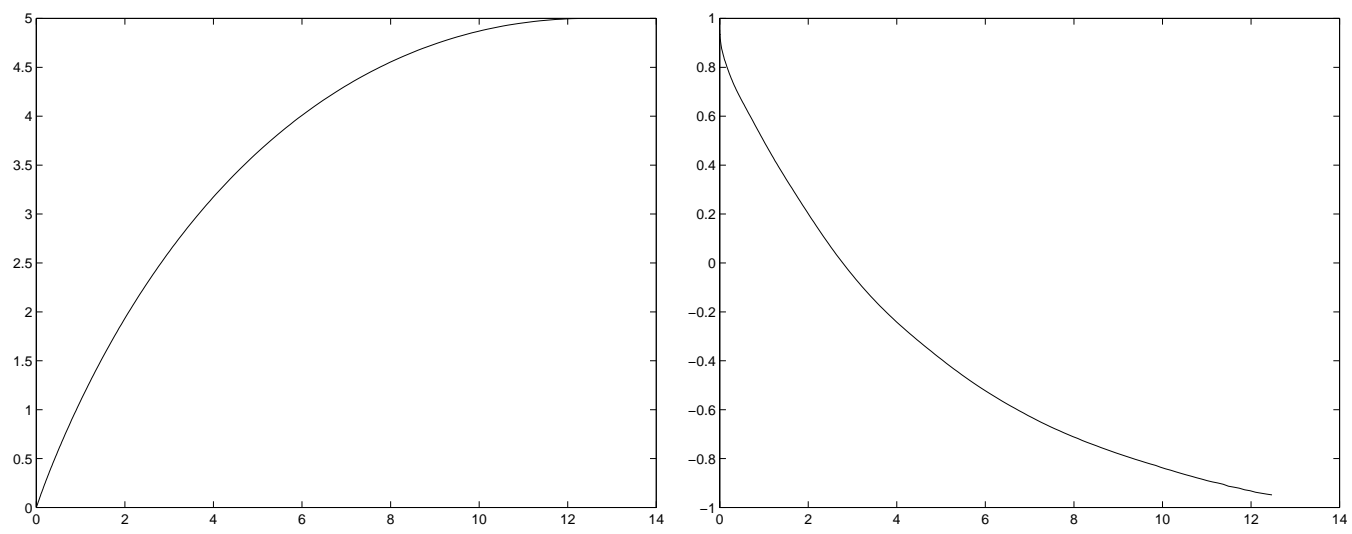

Figure 9.1: Height and control as a function of position for the particle steering problem 


\section{Goddard Rocket}

Maximize the final altitude of a vertically launched rocket, using the thrust as a control and given the initial mass, the fuel mass, and the drag characteristics of the rocket.

\section{Formulation}

This is a classical problem in dynamic optimization that is typical of control problems with a singular arc. See Bryson [7, pages 392-394] for background information. The equations of motion for the rocket are

$$
h^{\prime}=v, \quad v^{\prime}=\frac{T-D(h, v)}{m}-g(h), \quad m^{\prime}=-\frac{T}{c},
$$

where $h$ is the altitude from the center of the earth, $v$ is the vertical velocity, $T$ is the rocket thrust, $D$ is the aerodynamic drag, $g$ is the gravitational force, and $c$ is a constant that measures the impulse of the rocket fuel. The thrust must satisfy

$$
0 \leq T(t) \leq T_{\max }
$$

The drag and the gravitational force are defined by

$$
D(h, v)=D_{c} v^{2} \exp \left(-h_{c}\left(\frac{h-h(0)}{h(0)}\right)\right), \quad g(h)=g_{0}\left(\frac{h(0)}{h}\right)^{2},
$$

where $D_{c}$ and $h_{c}$ are constants, and $g_{0}$ is the gravitational force at the earth's surface. The rocket is initially at rest $(v(0)=0)$, and the mass at the end of the flight is a fraction of the initial mass,

$$
m\left(t_{f}\right)=m_{c} m(0),
$$

where $t_{f}$ is the flight time and $m_{c}$ is a constant. In addition to the bounds on the thrust, there are bounds

$$
m\left(t_{f}\right) \leq m(t) \leq m(0), \quad h(t) \geq h(0), \quad v(t) \geq 0,
$$

on the mass, altitude, and velocity of the rocket. These bounds are a direct consequence of the equations of motion (10.1).

The equations of motion can be made dimension free by scaling the equations and choosing the model parameters in terms of $h(0), m(0)$, and $g_{0}$. We follow [7] and use

$$
T_{\max }=3.5 g_{0} m(0), \quad D_{c}=\frac{1}{2} v_{c} \frac{m(0)}{g_{0}}, \quad c=\frac{1}{2}\left(g_{0} h(0)\right)^{1 / 2} .
$$

With these choices we can assume, without loss of generality, that $h(0)=m(0)=g_{0}=1$. We also follow [7] and choose

$$
h_{c}=500, \quad m_{c}=0.6, \quad v_{c}=620 .
$$

We discretize the equations of motion with the trapezoidal rule, and a uniform mesh with $n_{h}$ intervals. Data for this problem appears in Table 10.1. 
Table 10.1: Goddard rocket problem data

\begin{tabular}{|l|c|}
\hline Variables & $4 n_{h}+1$ \\
Constraints & $3 n_{h}$ \\
Bounds & $4 n_{h}+1$ \\
Linear equality constraints & 0 \\
Linear inequality constraints & 0 \\
Nonlinear equality constraints & $3 n_{h}$ \\
Nonlinear inequality constraints & 0 \\
Nonzeros in $\nabla^{2} f(x)$ & 0 \\
Nonzeros in $c^{\prime}(x)$ & $19 n_{h}-8$ \\
\hline
\end{tabular}

\section{Performance}

Results for the AMPL implementation are shown in Table 10.2. For starting points we use $t_{f}=1$ and the functions $h=1$,

$$
v(t)=\frac{t}{t_{f}}\left(1-\frac{t}{t_{f}}\right), \quad m(t)=\left(m_{f}-m_{0}\right)\left(\frac{t}{t_{f}}\right)+m_{0},
$$

evaluated at the grid points. The initial value for the thrust is $T=T_{\max } / 2$.

Table 10.2: Performance on Goddard rocket problem

\begin{tabular}{|c|c|c|c|}
\hline Solver & $n_{h}=400$ & $n_{h}=800$ & $n_{h}=1600$ \\
\hline \hline FILTER & $10.58 \mathrm{~s}$ & $\ddagger$ & $263.83 \mathrm{~s}$ \\
$f$ & $1.01283 \mathrm{e}+00$ & $\ddagger$ & $1.01283 \mathrm{e}+00$ \\
$c$ violation & $4.20 \mathrm{e}-13$ & $\ddagger$ & $8.93 \mathrm{e}-14$ \\
optimality & $4.55 \mathrm{e}-11$ & $\ddagger$ & $8.11 \mathrm{e}-12$ \\
\hline KNITRO & $7.61 \mathrm{~s}$ & $74.97 \mathrm{~s}$ & $309.73 \mathrm{~s}$ \\
$f$ & $1.01283 \mathrm{e}+00$ & $1.01283 \mathrm{e}+00$ & $1.01283 \mathrm{e}+00$ \\
$c$ violation & $3.75 \mathrm{e}-08$ & $1.54 \mathrm{e}-08$ & $7.03 \mathrm{e}-08$ \\
optimality & $1.94 \mathrm{e}-07$ & $3.47 \mathrm{e}-08$ & $4.40 \mathrm{e}-08$ \\
\hline LOQO & $1.55 \mathrm{~s}$ & $3.43 \mathrm{~s}$ & $7.64 \mathrm{~s}$ \\
$f$ & $1.01283 \mathrm{e}+00$ & $1.01283 \mathrm{e}+00$ & $1.01283 \mathrm{e}+00$ \\
$c$ violation & $3.06 \mathrm{e}-09$ & $4.92 \mathrm{e}-10$ & $2.40 \mathrm{e}-09$ \\
optimality & $2.60 \mathrm{e}-08$ & $4.17 \mathrm{e}-09$ & $3.88 \mathrm{e}-09$ \\
\hline MINOS & $0.36 \mathrm{~s}$ & $1.95 \mathrm{~s}$ & $4.27 \mathrm{~s}$ \\
$f$ & $6.11246 \mathrm{e}+03 \dagger$ & $5.83895 \mathrm{e}+07 \dagger$ & $6.86120 \mathrm{e}+07 \dagger$ \\
$c$ violation & $1.00 \mathrm{e}+00 \dagger$ & $1.00 \mathrm{e}+00 \dagger$ & $1.00 \mathrm{e}+00 \dagger$ \\
optimality & $1.00 \mathrm{e}+00 \dagger$ & $1.00 \mathrm{e}+00 \dagger$ & $1.00 \mathrm{e}+00 \dagger$ \\
\hline SNOPT & $13.21 \mathrm{~s}$ & $37.64 \mathrm{~s}$ & $143.38 \mathrm{~s}$ \\
$f$ & $1.01282 \mathrm{e}+00$ & $1.01283 \mathrm{e}+00$ & $1.01283 \mathrm{e}+00$ \\
$c$ violation & $3.35 \mathrm{e}-08$ & $1.02 \mathrm{e}-08$ & $6.71 \mathrm{e}-08$ \\
optimality & $5.29 \mathrm{e}-07$ & $1.31 \mathrm{e}-07$ & $2.76 \mathrm{e}-07$ \\
\hline
\end{tabular}

$\dagger$ Errors. $\ddagger$ Timed out. $\uparrow$ Failed benchmark tests.

Figure 10.1 shows the altitude and mass of the rocket as a function of time. Note that altitude increases until a maximum altitude of $h=1.01$ is reached, while the mass of the rocket steadily decreases until the final mass of $m\left(t_{f}\right)=0.6$ is reached at $t=0.073$. 
Figure 10.2 shows the velocity and thrust as a function of time. The thrust is bangsingular-bang, with the region of singularity occurring when

$$
0<T(t)<T_{\max } .
$$

This figure shows that the optimal flight path involves using maximal thrust until $t=0.022$, and no thrust for $t \geq 0.073$, at which point the final mass is reached, and the rocket coasts to its maximal altitude. The oscillations that appear at the point of discontinuity in the thrust parameter can be removed by using more grid points.
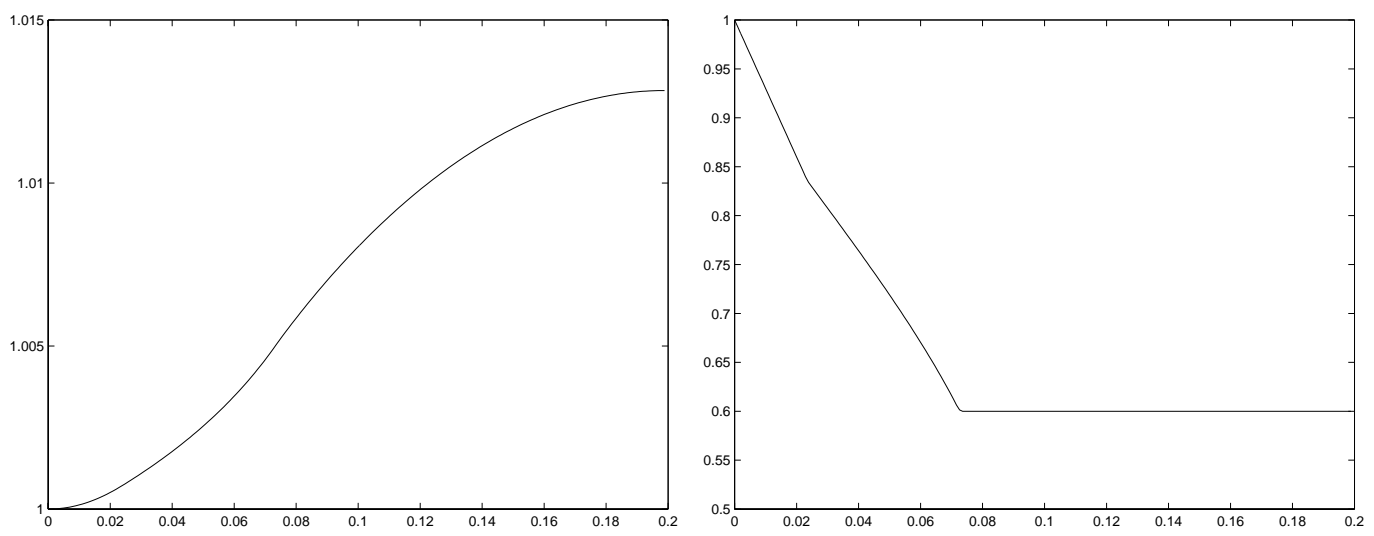

Figure 10.1: Altitude and mass for the Goddard rocket problem
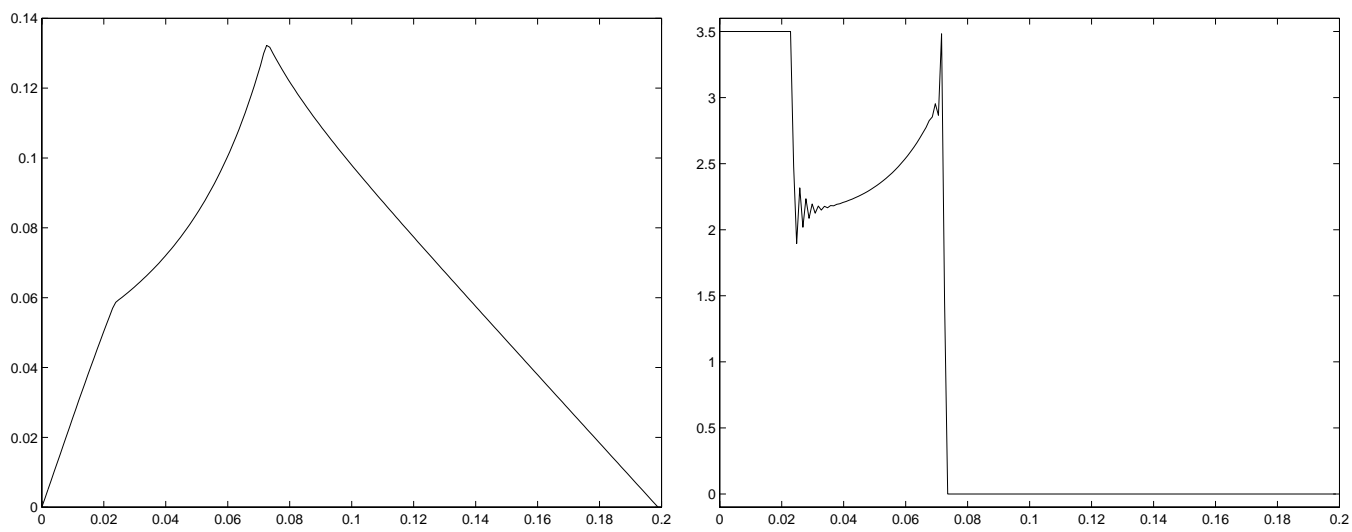

Figure 10.2: Velocity and thrust for the Goddard rocket problem 


\section{Hang Glider}

Maximize the final horizontal position of a hang glider while in the presence of a thermal updraft.

\section{Formulation}

The formulation of this problem follows [8]. The equations of motion for the hang glider are

$$
x^{\prime \prime}=\frac{1}{m}(-L \sin (\eta)-D \cos (\eta)), \quad y^{\prime \prime}=\frac{1}{m}(L \cos (\eta)-D \sin (\eta))-g,
$$

where $(x, y)$ is the position of the glider, $m$ is the mass of the glider, $g$ is the gravitational constant, and the function $\eta$ is defined by

$$
\sin (\eta)=\frac{w\left(x, y^{\prime}\right)}{v\left(x, x^{\prime}, y^{\prime}\right)}, \quad \cos (\eta)=\frac{x^{\prime}}{v\left(x, x^{\prime}, y^{\prime}\right)},
$$

where

$$
\begin{gathered}
v\left(x, x^{\prime}, y^{\prime}\right)=\sqrt{x^{\prime 2}+w\left(x, y^{\prime}\right)^{2}}, \quad w\left(x, y^{\prime}\right)=y^{\prime}-u(x), \\
u(x)=u_{c}(1-r(x)) \exp (-r(x)), \quad r(x)=\left(\frac{x}{r_{c}}-2.5\right)^{2},
\end{gathered}
$$

and constants $u_{c}=2.5$ and $r_{c}=100$. The updraft function $u$ is positive in a neighborhood of $x=2.5 r_{c}$ but drops to zero exponentially away from $x=2.5 r_{c}$. The functions $D$ and $L$ are defined by

$$
D\left(x, x^{\prime}, y^{\prime}, c_{L}\right)=\frac{1}{2}\left(c_{0}+c_{1} c_{L}^{2}\right) \rho S v\left(x, x^{\prime}, y^{\prime}\right)^{2}, \quad L\left(x, x^{\prime}, y^{\prime}, c_{L}\right)=\frac{1}{2} c_{L} \rho S v\left(x, x^{\prime}, y^{\prime}\right)^{2},
$$

where $S$ is the wing area, $\rho$ is the air density, $c_{L}$ is the aerodynamic lift coefficient, and $c_{0}+c_{1} c_{L}^{2}$ is the drag coefficient. For this glider

$$
c_{0}=0.034, \quad c_{1}=0.069662, \quad S=14, \quad \rho=1.13 .
$$

The aerodynamic lift coefficient $c_{L}$ must satisfy the bounds

$$
0 \leq c_{L}(t) \leq c_{\max }
$$

and we also impose the natural bounds $x \geq 0$ and $x^{\prime} \geq 0$. In this problem $c_{\max }=1.4$, $m=100, g=9.81$, and the boundary conditions are

$$
\begin{gathered}
x(0)=0, \quad y(0)=1000, \quad y\left(t_{f}\right)=900, \\
x^{\prime}(0)=x^{\prime}\left(t_{f}\right)=13.23, \quad y^{\prime}(0)=y^{\prime}\left(t_{f}\right)=-1.288 .
\end{gathered}
$$

Discretization is done with a uniform time step and the trapezoidal rule over $n_{h}$ intervals. Data for this problem is shown in Table 11.1. 
Table 11.1: Hang glider problem data

\begin{tabular}{|l|c|}
\hline Variables & $5 n_{h}-1$ \\
Constraints & $4 n_{h}$ \\
Bounds & $3 n_{h}+1$ \\
Linear equality constraints & 0 \\
Linear inequality constraints & 0 \\
Nonlinear equality constraints & $4 n_{h}$ \\
Nonlinear inequality constraints & 0 \\
Nonzeros in $\nabla^{2} f(x)$ & 0 \\
Nonzeros in $c^{\prime}(x)$ & $28 n_{h}-17$ \\
\hline
\end{tabular}

\section{Performance}

Results for the AMPL implementation are shown in Table 11.2. For starting points we use $t_{f}=1$ and the functions $x^{\prime}=x^{\prime}(0), y^{\prime}=y^{\prime}(0)$, and

$$
x(t)=x(0)+x^{\prime}(0)\left(\frac{t}{t_{f}}\right), \quad y(t)=y(0)+\left(y\left(t_{f}\right)-y(0)\right)\left(\frac{t}{t_{f}}\right),
$$

evaluated at the grid points. The initial value for the control is $c_{L}(t)=\frac{1}{2} c_{\max }$.

Table 11.2: Performance on hang glider problem

\begin{tabular}{|c|c|c|c|}
\hline Solver & $n_{h}=100$ & $n_{h}=200$ & $n_{h}=400$ \\
\hline \hline FILTER & $121.89 \mathrm{~s}$ & $521.66 \mathrm{~s}$ & $\ddagger$ \\
$f$ & $1.25461 \mathrm{e}+03$ & $1.24880 \mathrm{e}+03$ & $\ddagger$ \\
$c$ violation & $2.27 \mathrm{e}-13$ & $2.27 \mathrm{e}-13$ & $\ddagger$ \\
optimality & $2.08 \mathrm{e}-07$ & $6.46 \mathrm{e}-08$ & $\ddagger$ \\
\hline KNITRO & $132.43 \mathrm{~s}$ & $932.48 \mathrm{~s}$ & $575.8 \mathrm{~s}$ \\
$f$ & $1.25505 \mathrm{e}+03$ & $1.24880 \mathrm{e}+03$ & $1.24797 \mathrm{e}+03$ \\
$c$ violation & $3.23 \mathrm{e}-09$ & $1.56 \mathrm{e}-10$ & $1.20 \mathrm{e}-10$ \\
optimality & $7.74 \mathrm{e}-07$ & $4.21 \mathrm{e}-08$ & $9.24 \mathrm{e}-07$ \\
\hline LOQO & $\ddagger$ & $202.48 \mathrm{~s}$ & $\ddagger$ \\
$f$ & $\ddagger$ & $1.24889 \mathrm{e}+03$ & $\ddagger$ \\
$c$ violation & $\ddagger$ & $2.64 \mathrm{e}-09$ & $\ddagger$ \\
optimality & $\ddagger$ & $3.12 \mathrm{e}-09$ & $\ddagger$ \\
\hline MINOS & $20.16 \mathrm{~s}$ & $20.3 \mathrm{~s}$ & $49.1 \mathrm{~s}$ \\
$f$ & $1.45505 \mathrm{e}+04 \dagger$ & $6.66929 \mathrm{e}+03 \dagger$ & $1.25819 \mathrm{e}+04 \dagger$ \\
$c$ violation & $1.00 \mathrm{e}+00 \dagger$ & $1.00 \mathrm{e}+00 \dagger$ & $1.00 \mathrm{e}+00 \dagger$ \\
optimality & $1.00 \mathrm{e}+00 \dagger$ & $1.00 \mathrm{e}+00 \dagger$ & $1.00 \mathrm{e}+00 \dagger$ \\
\hline SNOPT & $39.1 \mathrm{~s}$ & $176.52 \mathrm{~s}$ & $345.13 \mathrm{~s}$ \\
$f$ & $1.25505 \mathrm{e}+03$ & $2.38432 \mathrm{e}+05 \dagger$ & $2.06594 \mathrm{e}+03 \dagger$ \\
$c$ violation & $3.06 \mathrm{e}-12$ & $1.00 \mathrm{e}+00 \dagger$ & $1.00 \mathrm{e}+00 \dagger$ \\
optimality & $9.09 \mathrm{e}-07$ & $1.00 \mathrm{e}+00 \dagger$ & $5.00 \mathrm{e}-01 \dagger$ \\
\hline
\end{tabular}

$\dagger$ Errors. $\ddagger$ Timed out. $\uparrow$ Failed benchmark tests.

Figure 11.1 shows the altitude and control function $c_{L}$ as a function of time. The glider starts at an altitude of $y(0)=1000$ and descends until the glider meets the updraft centered 
at $x=250$. As a result the glider climbs and then descends to the desired final altitude of $y\left(t_{f}\right)=900$ at time $t_{f}=105$.
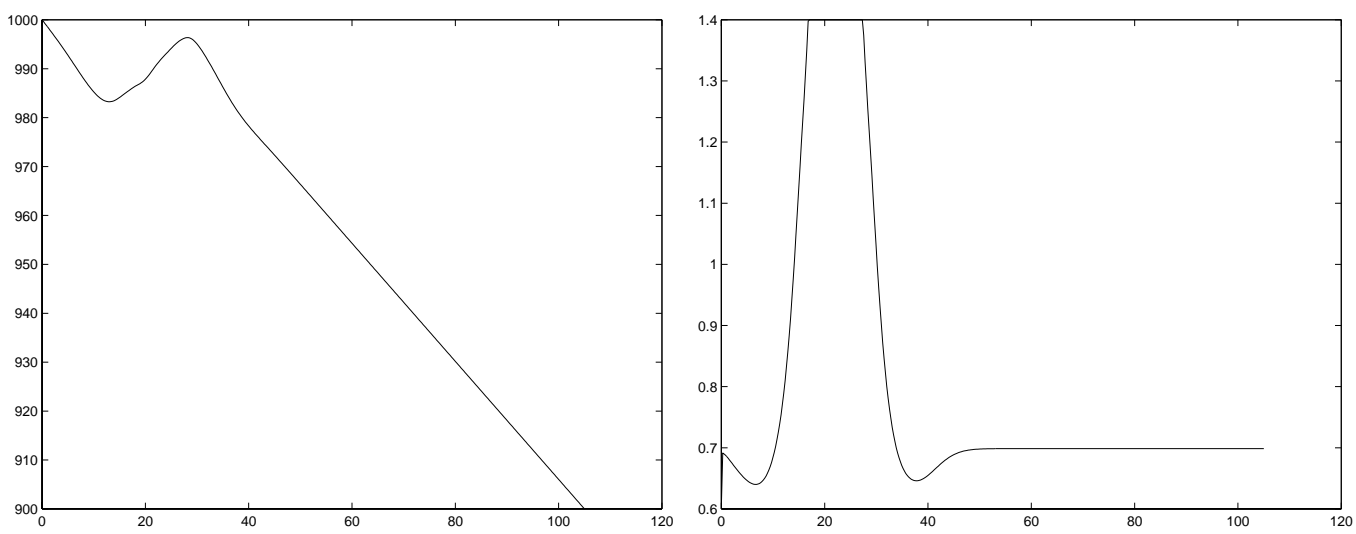

Figure 11.1: Altitude and control $c_{L}$ for the hang glider problem

Figure 11.2 shows velocities $x^{\prime}$ and $y^{\prime}$ as a function of time. Note, in particular, the erratic behavior of the velocities while the control is in the bang-region where $c_{L}(t)=c_{\max }$.
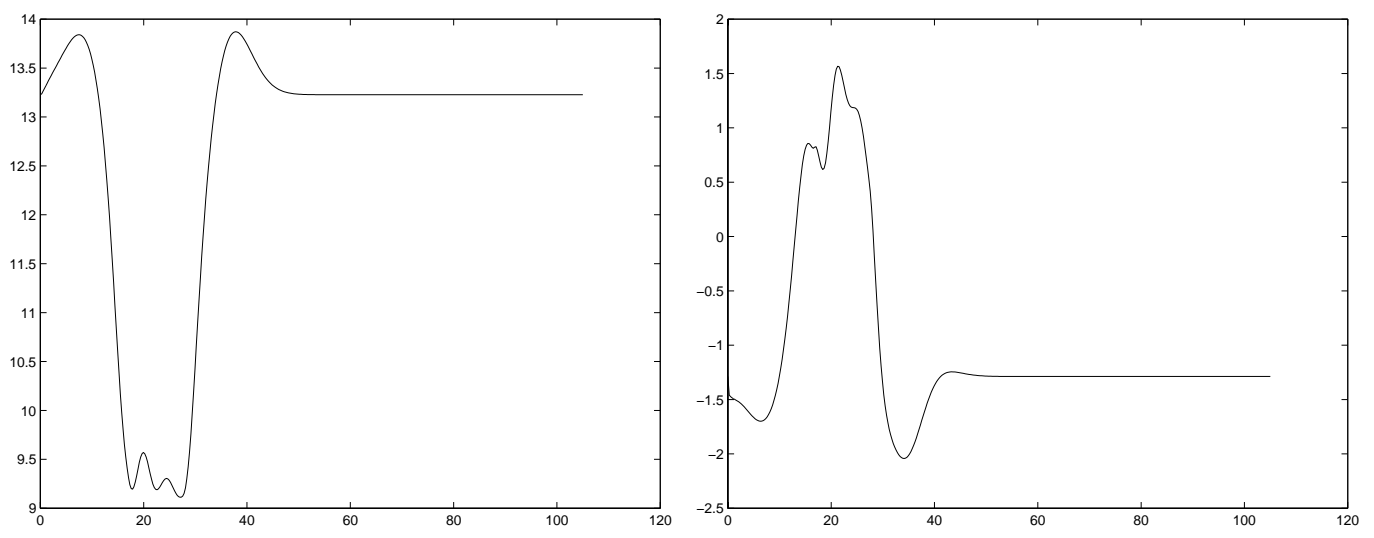

Figure 11.2: Velocities $x^{\prime}$ and $y^{\prime}$ for the hang glider problem 


\section{Catalytic Cracking of Gas Oil}

Determine the reaction coefficients for the catalytic cracking of gas oil into gas and other byproducts. The nonlinear model [31] that describes the process is

$$
\begin{aligned}
& y_{1}^{\prime}=-\left(\theta_{1}+\theta_{3}\right) y_{1}^{2} \\
& y_{2}^{\prime}=\theta_{1} y_{1}^{2}-\theta_{2} y_{2}
\end{aligned}
$$

with coefficients $\theta_{i} \geq 0$ for $i=1, \ldots, 3$. Initial conditions for (12.1) are known. The problem is to minimize

$$
\sum_{j=1}^{21}\left\|y\left(\tau_{j} ; \theta\right)-z_{j}\right\|^{2}
$$

where $z_{j}$ are concentration measurements for $y$ at time points $\tau_{1}, \ldots, \tau_{21}$.

\section{Formulation}

Our formulation of the catalytic cracking of gas oil problem as an optimization problem follows $[31,3]$. We use a $k$-stage collocation method, a uniform partition of the interval $\left[0, \tau_{21}\right]$ with $n_{h}$ intervals, and the standard [2, pages 247-249] basis representation,

$$
p_{\pi}(t)=v_{i}+\sum_{j=1}^{k} \frac{\left(t-t_{i}\right)^{j}}{j ! h^{j-1}} w_{i j}, \quad t \in\left[t_{i}, t_{i+1}\right],
$$

for the components of the solution $\left(y_{1}, y_{2}\right)$ of $(12.1)$. The constraints in the optimization problem are the initial conditions in (12.1), the continuity conditions, and the collocation equations. The continuity equations are a set of $2\left(n_{h}-1\right)$ linear equations. The collocation equations are a set of $2 k n_{h}$ nonlinear equations obtained by requiring that the collocation approximation satisfy (12.1) at the collocation points. Data for this problem appears in Table 12.1. The number of nonzeros in the Hessian of the objective function is an upper bound. Fewer nonzeros are present if the times at which the measurements were taken coincide with the grid points.

Table 12.1: Catalytic cracking of gas oil data

\begin{tabular}{|l|c|}
\hline Variables & $2(k+1) n_{h}+1$ \\
Constraints & $2(k+1) n_{h}-2$ \\
Bounds & 3 \\
Linear equality constraints & $2\left(n_{h}-1\right)$ \\
Linear inequality constraints & 0 \\
Nonlinear equality constraints & $2 k n_{h}$ \\
Nonlinear inequality constraints & 0 \\
Nonzeros in $\nabla^{2} f(x)$ & $42(k+1)^{2}$ \\
Nonzeros in $c^{\prime}(x)$ & $3 k(k+3) n_{h}+4 n_{h}-5 k-6$ \\
\hline
\end{tabular}




\section{Performance}

We provide results for the AMPL formulation with $k=4$ in Table 12.2. The initial values for the $\theta$ parameters are $\theta_{i}=0.0$. The initial basis parameters are chosen so that the collocation approximation is piecewise constant and interpolates the data. Data is generated by solving (12.1) numerically using the Tjoa and Biegler [31] values $\theta=(12,8,2)$ and applying a relative random perturbation of size $10^{-1}$. Figure 12.1 shows the solution and the data.

Table 12.2: Performance on catalytic cracking of gas oil problem

\begin{tabular}{|c|c|c|c|}
\hline Solver & $n_{h}=100$ & $n_{h}=200$ & $n_{h}=400$ \\
\hline \hline FILTER & $2.86 \mathrm{~s}$ & $13.61 \mathrm{~s}$ & $68.84 \mathrm{~s}$ \\
$f$ & $5.23659 \mathrm{e}-03$ & $5.23659 \mathrm{e}-03$ & $5.23659 \mathrm{e}-03$ \\
$c$ violation & $2.87 \mathrm{e}-15$ & $3.79 \mathrm{e}-09$ & $2.96 \mathrm{e}-09$ \\
optimality & $4.64 \mathrm{e}-11$ & $2.00 \mathrm{e}-10$ & $1.48 \mathrm{e}-10$ \\
\hline KNITRO & $1.75 \mathrm{~s}$ & $5.52 \mathrm{~s}$ & $17.82 \mathrm{~s}$ \\
$f$ & $5.23659 \mathrm{e}-03$ & $5.23659 \mathrm{e}-03$ & $5.23659 \mathrm{e}-03$ \\
$c$ violation & $4.03 \mathrm{e}-10$ & $2.51 \mathrm{e}-09$ & $3.90 \mathrm{e}-08$ \\
optimality & $2.91 \mathrm{e}-08$ & $1.19 \mathrm{e}-09$ & $5.80 \mathrm{e}-08$ \\
\hline LOQO & $0.4 \mathrm{~s}$ & $0.99 \mathrm{~s}$ & $3.07 \mathrm{~s}$ \\
$f$ & $5.23659 \mathrm{e}-03$ & $5.23659 \mathrm{e}-03$ & $5.23659 \mathrm{e}-03$ \\
$c$ violation & $3.35 \mathrm{e}-08$ & $1.87 \mathrm{e}-08$ & $3.23 \mathrm{e}-08$ \\
optimality & $8.85 \mathrm{e}-11$ & $2.16 \mathrm{e}-10$ & $3.42 \mathrm{e}-10$ \\
\hline MINOS & $1.85 \mathrm{~s}$ & $6.42 \mathrm{~s}$ & $18.69 \mathrm{~s}$ \\
$f$ & $5.23659 \mathrm{e}-03$ & $5.23659 \mathrm{e}-03$ & $5.23659 \mathrm{e}-03$ \\
$c$ violation & $9.34 \mathrm{e}-12$ & $1.27 \mathrm{e}-08$ & $5.93 \mathrm{e}-09$ \\
optimality & $3.14 \mathrm{e}-11$ & $4.68 \mathrm{e}-10$ & $2.96 \mathrm{e}-10$ \\
\hline SNOPT & $2.41 \mathrm{~s}$ & $8.88 \mathrm{~s}$ & $25.54 \mathrm{~s}$ \\
$f$ & $5.23659 \mathrm{e}-03$ & $5.23659 \mathrm{e}-03$ & $5.23659 \mathrm{e}-03$ \\
$c$ violation & $4.81 \mathrm{e}-09$ & $8.22 \mathrm{e}-09$ & $7.20 \mathrm{e}-08$ \\
optimality & $2.48 \mathrm{e}-08$ & $1.44 \mathrm{e}-08$ & $2.24 \mathrm{e}-09$ \\
\hline
\end{tabular}

$\dagger$ Errors. †Timed out. $₫$ Failed benchmark tests.

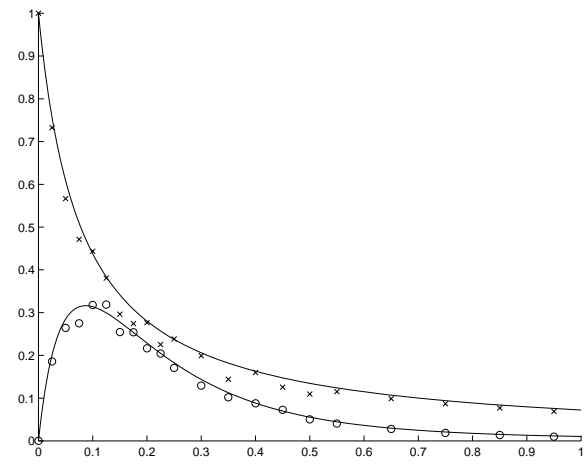

Figure 12.1: Solution and data for the catalytic cracking of gas oil problem 


\section{Methanol to Hydrocarbons}

Determine the reaction coefficients for the conversion of methanol into various hydrocarbons. The nonlinear model $[14,22]$ that describes the process is

$$
\begin{aligned}
y_{1}^{\prime} & =-\left(2 \theta_{2}-\frac{\theta_{1} y_{2}}{\left(\theta_{2}+\theta_{5}\right) y_{1}+y_{2}}+\theta_{3}+\theta_{4}\right) y_{1} \\
y_{2}^{\prime} & =\frac{\theta_{1} y_{1}\left(\theta_{2} y_{1}-y_{2}\right)}{\left(\theta_{2}+\theta_{5}\right) y_{1}+y_{2}}+\theta_{3} y_{1} \\
y_{3}^{\prime} & =\frac{\theta_{1} y_{1}\left(y_{2}+\theta_{5} y_{1}\right)}{\left(\theta_{2}+\theta_{5}\right) y_{1}+y_{2}}+\theta_{4} y_{1}
\end{aligned}
$$

with coefficients $\theta_{i} \geq 0$. Initial conditions for (13.1) are known. The problem is to minimize

$$
\sum_{j=1}^{16}\left\|y\left(\tau_{j} ; \theta\right)-z_{j}\right\|^{2}
$$

where $z_{j}$ are concentration measurements for $y$ at time points $\tau_{1}, \ldots, \tau_{16}$.

\section{Formulation}

Our formulation of the methanol-to-hydrocarbons problem as an optimization problem follows $[31,3]$. We use a $k$-stage collocation method, a uniform partition of the interval $\left[0, \tau_{16}\right]$ with $n_{h}$ intervals, and the standard [2, pages 247-249] basis representation,

$$
p_{\pi}(t)=v_{i}+\sum_{j=1}^{k} \frac{\left(t-t_{i}\right)^{j}}{j ! h^{j-1}} w_{i j}, \quad t \in\left[t_{i}, t_{i+1}\right],
$$

for the components of the solution $\left(y_{1}, y_{2}, y_{3}\right)$ of (13.1). The constraints in the optimization problem are the initial conditions in (13.1), the continuity conditions, and the collocation equations. The continuity equations are a set of $3\left(n_{h}-1\right)$ linear equations. The collocation equations are a set of $3 k n_{h}$ nonlinear equations obtained by requiring that the collocation approximation satisfy (13.1) at the collocation points. Data for this problem appears in Table 13.1.

Table 13.1: Methanol-to-hydrocarbons data

\begin{tabular}{|l|c|}
\hline Variables & $3(k+1) n_{h}+2$ \\
Constraints & $3(k+1) n_{h}-3$ \\
Bounds & 5 \\
Linear equality constraints & $3\left(n_{h}-1\right)$ \\
Linear inequality constraints & 0 \\
Nonlinear equality constraints & $3 k n_{h}$ \\
Nonlinear inequality constraints & 0 \\
Nonzeros in $\nabla^{2} f(x)$ & $48(k+1)^{2}$ \\
Nonzeros in $c^{\prime}(x)$ & $(7 k+22) k n_{h}+6 n_{h}-9 k-9$ \\
\hline
\end{tabular}


In Table 13.1, the number of nonzeros in the Hessian of the objective function is an upper bound. Fewer nonzeros are present if the times at which the measurements were taken coincide with the grid points.

\section{Performance}

We provide results for the AMPL formulation with $k=3$ in Table 13.2. The initial values for the $\theta$ parameters are $\theta_{i}=1.0$. The initial basis parameters are chosen so that the collocation approximation is piecewise constant and interpolates the data. Data is generated by solving (13.1) numerically using $\theta=(2.69,0.5,3.02,0.5,0.5)$ as given in Maria [22] and applying a relative random perturbation of size $10^{-1}$. Figure 13.1 shows the solution and the data.

Table 13.2: Performance on methanol-to-hydrocarbons problem

\begin{tabular}{|c|c|c|c|}
\hline Solver & $n_{h}=100$ & $n_{h}=200$ & $n_{h}=400$ \\
\hline \hline FILTER & $17.64 \mathrm{~s}$ & $85.31 \mathrm{~s}$ & $651.02 \mathrm{~s}$ \\
$f$ & $9.02229 \mathrm{e}-03$ & $9.02229 \mathrm{e}-03$ & $9.02229 \mathrm{e}-03$ \\
$c$ violation & $8.71 \mathrm{e}-15$ & $6.54 \mathrm{e}-11$ & $1.07 \mathrm{e}-12$ \\
optimality & $1.14 \mathrm{e}-14$ & $1.82 \mathrm{e}-13$ & $2.21 \mathrm{e}-13$ \\
\hline KNITRO & $1.95 \mathrm{~s}$ & $5.43 \mathrm{~s}$ & $9.01 \mathrm{~s}$ \\
$f$ & $9.02229 \mathrm{e}-03$ & $9.02229 \mathrm{e}-03$ & $9.02229 \mathrm{e}-03$ \\
$c$ violation & $2.38 \mathrm{e}-09$ & $2.41 \mathrm{e}-09$ & $2.42 \mathrm{e}-09$ \\
optimality & $2.77 \mathrm{e}-11$ & $1.71 \mathrm{e}-11$ & $9.13 \mathrm{e}-12$ \\
\hline LOQO & $0.86 \mathrm{~s}$ & $2.11 \mathrm{~s}$ & $5.85 \mathrm{~s}$ \\
$f$ & $9.02229 \mathrm{e}-03$ & $9.02229 \mathrm{e}-03$ & $9.02229 \mathrm{e}-03$ \\
$c$ violation & $7.54 \mathrm{e}-08$ & $2.64 \mathrm{e}-08$ & $2.07 \mathrm{e}-08$ \\
optimality & $5.65 \mathrm{e}-12$ & $3.30 \mathrm{e}-12$ & $5.01 \mathrm{e}-12$ \\
\hline MINOS & $2.17 \mathrm{~s}$ & $4.54 \mathrm{~s}$ & $44.85 \mathrm{~s}$ \\
$f$ & $9.02229 \mathrm{e}-03$ & $9.02229 \mathrm{e}-03$ & $9.02229 \mathrm{e}-03$ \\
$c$ violation & $5.80 \mathrm{e}-13$ & $2.32 \mathrm{e}-11$ & $3.00 \mathrm{e}-13$ \\
optimality & $1.02 \mathrm{e}-09$ & $9.20 \mathrm{e}-10$ & $9.07 \mathrm{e}-10$ \\
\hline SNOPT & $5.15 \mathrm{~s}$ & $14.53 \mathrm{~s}$ & $66.93 \mathrm{~s}$ \\
$f$ & $9.02229 \mathrm{e}-03$ & $9.02229 \mathrm{e}-03$ & $9.02229 \mathrm{e}-03$ \\
$c$ violation & $1.28 \mathrm{e}-10$ & $3.19 \mathrm{e}-10$ & $7.48 \mathrm{e}-11$ \\
optimality & $4.24 \mathrm{e}-08$ & $5.24 \mathrm{e}-08$ & $7.05 \mathrm{e}-09$ \\
\hline
\end{tabular}

$\dagger$ Errors. $\ddagger$ Timed out. $\uparrow$ Failed benchmark tests.

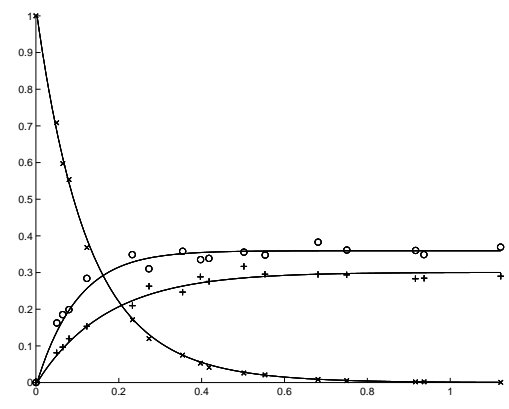

Figure 13.1: Solution and data for the methanol-to-hydrocarbons problem 


\section{Catalyst Mixing}

Determine the optimal mixing policy of two catalysts along the length of a tubular plug flow reactor involving several reactions.

\section{Formulation}

The nonlinear model [34] that describes the reactions is

$$
\begin{aligned}
& x_{1}^{\prime}(t)=u(t)\left(10 x_{2}(t)-x_{1}(t)\right) \\
& x_{2}^{\prime}(t)=u(t)\left(x_{1}(t)-10 x_{2}(t)\right)-(1-u(t)) x_{2}(t) .
\end{aligned}
$$

Initial conditions for (14.1) are $x_{1}(0)=1$ and $x_{2}(0)=0$. The control variable $u$ represents the mixing ratio of the catalysts and must satisfy the bounds

$$
0 \leq u(t) \leq 1
$$

The problem is to minimize

$$
-1+x_{1}\left(t_{f}\right)+x_{2}\left(t_{f}\right), \quad t_{f}=1 \text {. }
$$

We formulate this problem with a $k$-stage collocation method, a uniform partition of the interval $[0,1]$ with $n_{h}$ intervals, and the standard [2, pages 247-249] basis representation,

$$
p_{\pi}(t)=v_{i}+\sum_{j=1}^{k} \frac{\left(t-t_{i}\right)^{j}}{j ! h^{j-1}} w_{i j}, \quad t \in\left[t_{i}, t_{i+1}\right],
$$

for the components of the solution of (14.1). The constraints in the optimization problem are the initial conditions in (14.1), the continuity conditions, and the collocation equations. Data for this problem appears in Table 14.1.

Table 14.1: Catalyst mixing data

\begin{tabular}{|l|c|}
\hline Variables & $(3 k+2) n_{h}-2$ \\
Constraints & $(2 k+2) n_{h}-2$ \\
Bounds & $k n_{h}$ \\
Linear equality constraints & $2\left(n_{h}-1\right)$ \\
Linear inequality constraints & 0 \\
Nonlinear equality constraints & $2 k n_{h}$ \\
Nonlinear inequality constraints & 0 \\
Nonzeros in $\nabla^{2} f(x)$ & 0 \\
Nonzeros in $c^{\prime}(x)$ & $(4 k+8) k n_{h}+4 n_{h}-6 k-6$ \\
\hline
\end{tabular}

\section{Performance}

We provide results for the AMPL formulation with $k=3$ in Table 14.1. For starting points we use $u=0, x_{1}=1$, and $x_{2}=0$ evaluated at the grid points. 
The catalyst mixing problem is a typical bang-singular-bang problem. The singularity leads to nonunique values of the control in the singular region, and thus it is possible to obtain different values for the control. Figure 14.1 shows the controls obtained by two different solvers.

Table 14.2: Performance on catalyst mixing problem

\begin{tabular}{|c|c|c|c|}
\hline Solver & $n_{h}=100$ & $n_{h}=200$ & $n_{h}=400$ \\
\hline \hline FILTER & $21.63 \mathrm{~s}$ & $29.87 \mathrm{~s}$ & $500.74 \mathrm{~s}$ \\
$f$ & $-4.80556 \mathrm{e}-02$ & $-4.80556 \mathrm{e}-02$ & $-4.80556 \mathrm{e}-02$ \\
$c$ violation & $4.80 \mathrm{e}-10$ & $2.01 \mathrm{e}-08$ & $1.27 \mathrm{e}-09$ \\
optimality & $8.90 \mathrm{e}-10$ & $1.70 \mathrm{e}-09$ & $5.58 \mathrm{e}-10$ \\
\hline KNITRO & $9.06 \mathrm{~s}$ & $7.09 \mathrm{~s}$ & $7.37 \mathrm{~s}$ \\
$f$ & $-4.80556 \mathrm{e}-02$ & $-4.80556 \mathrm{e}-02$ & $-4.80556 \mathrm{e}-02 \dagger$ \\
$c$ violation & $8.10 \mathrm{e}-08$ & $1.76 \mathrm{e}-14$ & $3.36 \mathrm{e}-16 \dagger$ \\
optimality & $2.20 \mathrm{e}-07$ & $2.76 \mathrm{e}-07$ & $3.40 \mathrm{e}-08 \dagger$ \\
\hline LOQO & $3.57 \mathrm{~s}$ & $2 \mathrm{~s}$ & $19.22 \mathrm{~s}$ \\
$f$ & $-4.80556 \mathrm{e}-02$ & $-4.80556 \mathrm{e}-02$ & $-4.80556 \mathrm{e}-02 \dagger$ \\
$c$ violation & $6.16 \mathrm{e}-09$ & $2.11 \mathrm{e}-08$ & $2.88 \mathrm{e}-11 \dagger$ \\
optimality & $2.49 \mathrm{e}-09$ & $3.96 \mathrm{e}-09$ & $1.40 \mathrm{e}-11 \dagger$ \\
\hline MINOS & $8.88 \mathrm{~s}$ & $18.1 \mathrm{~s}$ & $32.93 \mathrm{~s}$ \\
$f$ & $-4.80538 \mathrm{e}-02$ & $-4.80534 \mathrm{e}-02$ & $-4.80477 \mathrm{e}-02$ \\
$c$ violation & $2.91 \mathrm{e}-08$ & $2.91 \mathrm{e}-08$ & $3.33 \mathrm{e}-08$ \\
optimality & $1.87 \mathrm{e}-07$ & $2.68 \mathrm{e}-07$ & $3.29 \mathrm{e}-07$ \\
\hline SNOPT & $9.3 \mathrm{~s}$ & $55.09 \mathrm{~s}$ & $179.14 \mathrm{~s}$ \\
$f$ & $-4.80555 \mathrm{e}-02$ & $-4.80556 \mathrm{e}-02$ & $-4.80516 \mathrm{e}-02$ \\
$c$ violation & $6.33 \mathrm{e}-08$ & $4.14 \mathrm{e}-08$ & $5.14 \mathrm{e}-11$ \\
optimality & $1.46 \mathrm{e}-07$ & $9.53 \mathrm{e}-09$ & $2.99 \mathrm{e}-07$ \\
\hline
\end{tabular}

$\dagger$ Errors. $\ddagger$ Timed out. $\uparrow$ Failed benchmark tests.
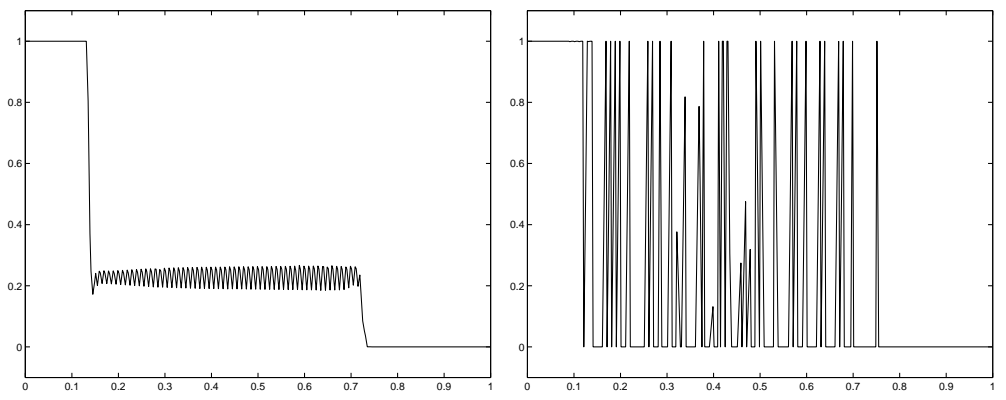

Figure 14.1: Controls obtained by two different solvers for the catalyst mixing problem 


\section{Elastic-Plastic Torsion}

Determine the stress potential in an infinitely long cylinder when torsion is applied.

\section{Formulation}

The elastic-plastic torsion problem [18, pages 41-46] can be formulated in terms of the cross-section $\mathcal{D}$ of the cylinder, and the torsion angle $c$ per unit length. The stress potential $u$ minimizes the quadratic $q: K \mapsto \mathbb{R}$,

$$
q(v)=\int_{\mathcal{D}}\left\{\frac{1}{2}\|\nabla v(x)\|^{2}-c v(x)\right\} d x,
$$

over the convex set $K$, where

$$
K=\left\{v \in H_{0}^{1}(\mathcal{D}):|v| \leq \operatorname{dist}(x, \partial \mathcal{D}), x \in \mathcal{D}\right\},
$$

$\operatorname{dist}(x, \partial \mathcal{D})$ is the distance from $x$ to the boundary of $\mathcal{D}$, and $H_{0}^{1}(\mathcal{D})$ is the space of functions with gradients in $L^{2}(\mathcal{D})$ that vanish on the boundary of $\mathcal{D}$.

A finite element approximation to the elastic-plastic torsion problem is obtained by triangulating $\mathcal{D}$ and minimizing $q$ over the space of piecewise linear functions with values $v_{i, j}$ at the vertices of the triangulation. We follow $[18,3]$ by choosing $\mathcal{D}=[0,1] \times[0,1]$, and using a triangulation with, respectively, $n_{x}$ and $n_{y}$ internal grid points in the coordinate directions. Data for this problem appears in Table 15.1.

Table 15.1: Elastic-plastic torsion problem data

\begin{tabular}{|l|c|}
\hline Variables & $n_{x} n_{y}$ \\
Constraints & 0 \\
Bounds & $n_{x} n_{y}$ \\
Linear equality constraints & 0 \\
Linear inequality constraints & 0 \\
Nonlinear equality constraints & 0 \\
Nonlinear inequality constraints & 0 \\
Nonzeros in $\nabla^{2} f(x)$ & $5 n_{x} n_{y}-2\left(n_{x}+n_{y}\right)$ \\
Nonzeros in $c^{\prime}(x)$ & 0 \\
\hline
\end{tabular}

\section{Performance}

We provide results for the AMPL formulation with $c=5$ in Table 15.2. For these results we fix $n_{x}=50$ and vary $n_{y}$. The starting guess is the function $\operatorname{dist}(x, \partial \mathcal{D})$ evaluated at the grid nodes. Figure 15.1 shows the potential in the torsion problem with $c=5$. The number of active constraints in this problem increases with $c$. Also

$$
\lim _{c \rightarrow \infty} v_{c}(x)=\operatorname{dist}(x, \partial \mathcal{D}),
$$

where $v_{c}$ is the potential as a function of $c$. 
Table 15.2: Performance on elastic-plastic torsion problem

\begin{tabular}{|c|c|c|c|}
\hline Solver & $n_{y}=50$ & $n_{y}=75$ & $n_{y}=100$ \\
\hline \hline FILTER & $135.92 \mathrm{~s}$ & $446.87 \mathrm{~s}$ & $545.44 \mathrm{~s}$ \\
$f$ & $-4.18087 \mathrm{e}-01$ & $-4.18199 \mathrm{e}-01$ & $-4.18239 \mathrm{e}-01$ \\
$c$ violation & $0.00 \mathrm{e}+00$ & $0.00 \mathrm{e}+00$ & $0.00 \mathrm{e}+00$ \\
optimality & $3.83 \mathrm{e}-07$ & $4.47 \mathrm{e}-07$ & $6.16 \mathrm{e}-07$ \\
\hline KNITRO & $258.79 \mathrm{~s}$ & $1415.58 \mathrm{~s}$ & $\ddagger$ \\
$f$ & $-4.18087 \mathrm{e}-01$ & $-4.18199 \mathrm{e}-01$ & $\ddagger$ \\
$c$ violation & $0.00 \mathrm{e}+00$ & $0.00 \mathrm{e}+00$ & $\ddagger$ \\
optimality & $5.82 \mathrm{e}-07$ & $4.77 \mathrm{e}-07$ & $\ddagger$ \\
\hline LOQO & $1.02 \mathrm{~s}$ & $1.72 \mathrm{~s}$ & $2.38 \mathrm{~s}$ \\
$f$ & $-4.18087 \mathrm{e}-01$ & $-4.18199 \mathrm{e}-01$ & $-4.18239 \mathrm{e}-01$ \\
$c$ violation & $0.00 \mathrm{e}+00$ & $0.00 \mathrm{e}+00$ & $0.00 \mathrm{e}+00$ \\
optimality & $2.50 \mathrm{e}-07$ & $1.59 \mathrm{e}-08$ & $9.34 \mathrm{e}-08$ \\
\hline MINOS & $59.98 \mathrm{~s}$ & $180.77 \mathrm{~s}$ & $402.23 \mathrm{~s}$ \\
$f$ & $-4.18087 \mathrm{e}-01$ & $-4.18199 \mathrm{e}-01$ & $-4.18239 \mathrm{e}-01$ \\
$c$ violation & $0.00 \mathrm{e}+00$ & $0.00 \mathrm{e}+00$ & $0.00 \mathrm{e}+00$ \\
optimality & $1.49 \mathrm{e}-07$ & $5.01 \mathrm{e}-07$ & $4.44 \mathrm{e}-07$ \\
\hline SNOPT & $12.36 \mathrm{~s}$ & $36.07 \mathrm{~s}$ & $91.02 \mathrm{~s}$ \\
$f$ & $-4.18087 \mathrm{e}-01$ & $-4.18199 \mathrm{e}-01$ & $-4.18239 \mathrm{e}-01$ \\
$c$ violation & $0.00 \mathrm{e}+00$ & $0.00 \mathrm{e}+00$ & $0.00 \mathrm{e}+00$ \\
optimality & $7.12 \mathrm{e}-15$ & $9.51 \mathrm{e}-15$ & $1.67 \mathrm{e}-14$ \\
\hline
\end{tabular}

$\dagger$ Errors. $\ddagger$ Timed out. $\uparrow$ Failed benchmark tests.

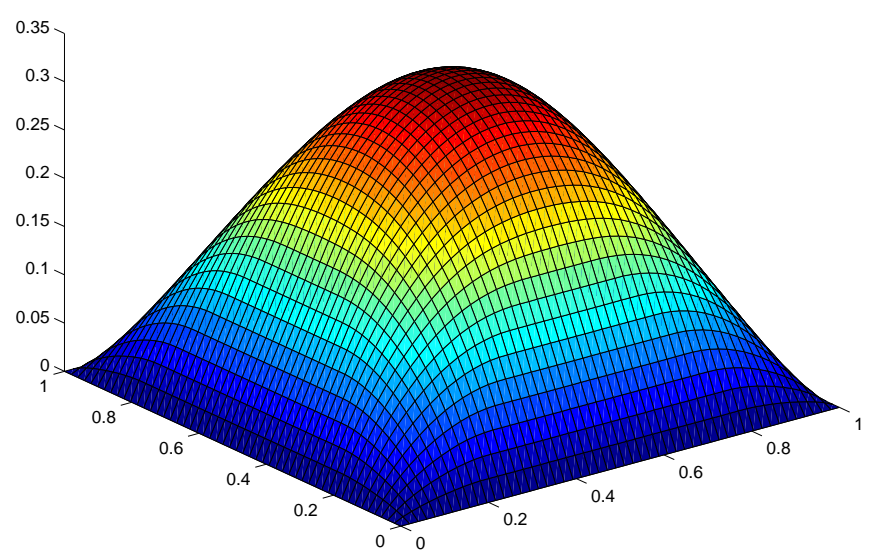

Figure 15.1: Elastic-plastic torsion problem with $c=5$ 


\section{Journal Bearing}

Given the eccentricity $\epsilon$ of the journal bearing, find the pressure distribution in the lubricant separating the shaft from the bearing.

\section{Formulation}

The journal bearing problem [9] requires determining the pressure between two circular cylinders of length $L$ and radii $R$ and $R+c$. The separation between the cylinders is $\varepsilon c$, where $\varepsilon$ is the eccentricity. The pressure $v$ minimizes the quadratic $q: K \mapsto \mathbb{R}$,

$$
q(v)=\int_{\mathcal{D}}\left\{\frac{1}{2} w_{q}(x)\|\nabla v(x)\|^{2}-w_{l}(x) v(x)\right\} d x
$$

over the convex set $K$, where $\mathcal{D}=(0,2 \pi) \times(0,2 b)$,

$$
K=\left\{v \in H_{0}^{1}(\mathcal{D}): v \geq 0\right\},
$$

$H_{0}^{1}(\mathcal{D})$ is the space of functions with gradients in $L^{2}(\mathcal{D})$ that vanish on the boundary of $\mathcal{D}$, and the functions $w_{q}: \mathcal{D} \mapsto \mathbb{R}$ and $w_{l}: \mathcal{D} \mapsto \mathbb{R}$ are defined by

$$
w_{q}\left(\xi_{1}, \xi_{2}\right)=\left(1+\varepsilon \cos \xi_{1}\right)^{3}, \quad w_{l}\left(\xi_{1}, \xi_{2}\right)=\varepsilon \sin \xi_{1},
$$

with $\varepsilon \in(0,1)$ the eccentricity of the bearing.

A finite element approximation to the journal bearing problem is obtained by triangulating $\mathcal{D}$ and minimizing $q$ over the space of piecewise linear functions with values $v_{i, j}$ at the vertices of the triangulation. We follow [3] by using a triangulation with, respectively, $n_{x}$ and $n_{y}$ internal grid points in the coordinate directions. Data for this problem appears in Table 16.1.

Table 16.1: Journal bearing problem data

\begin{tabular}{|l|c|}
\hline Variables & $n_{x} n_{y}$ \\
Constraints & 0 \\
Bounds & $n_{x} n_{y}$ \\
Linear equality constraints & 0 \\
Linear inequality constraints & 0 \\
Nonlinear equality constraints & 0 \\
Nonlinear inequality constraints & 0 \\
Nonzeros in $\nabla^{2} f(x)$ & $5 n_{x} n_{y}-2\left(n_{x}+n_{y}\right)$ \\
Nonzeros in $c^{\prime}(x)$ & 0 \\
\hline
\end{tabular}

\section{Performance}

We provide results with the AMPL formulation in Table 16.2 with $b=10$ and $\epsilon=0.1$. For these results we fix $n_{x}=50$ and vary $n_{y}$. The starting guess is the function $\max \{\sin (x), 0\}$ evaluated at the grid nodes. Figure 16.1 shows the pressure distribution for the journal bearing problem. 
Table 16.2: Performance on pressure in journal bearing problem

\begin{tabular}{|c|c|c|c|}
\hline Solver & $n_{y}=50$ & $n_{y}=75$ & $n_{y}=100$ \\
\hline \hline FILTER & $101.08 \mathrm{~s}$ & $312.17 \mathrm{~s}$ & $1058.45 \mathrm{~s}$ \\
$f$ & $-1.54824 \mathrm{e}-01$ & $-1.54984 \mathrm{e}-01$ & $-1.55042 \mathrm{e}-01$ \\
$c$ violation & $1.08 \mathrm{e}-18$ & $2.16 \mathrm{e}-19$ & $4.33 \mathrm{e}-19$ \\
optimality & $8.68 \mathrm{e}-07$ & $7.29 \mathrm{e}-07$ & $6.52 \mathrm{e}-07$ \\
\hline KNITRO & $101.83 \mathrm{~s}$ & $176.98 \mathrm{~s}$ & $477.6 \mathrm{~s}$ \\
$f$ & $-1.54824 \mathrm{e}-01$ & $-1.54984 \mathrm{e}-01 \dagger$ & $-1.55042 \mathrm{e}-01 \dagger$ \\
$c$ violation & $0.00 \mathrm{e}+00$ & $0.00 \mathrm{e}+00 \dagger$ & $0.00 \mathrm{e}+00 \dagger$ \\
optimality & $1.10 \mathrm{e}-08$ & $8.49 \mathrm{e}-09 \dagger$ & $6.21 \mathrm{e}-09 \dagger$ \\
\hline LOQO & $0.85 \mathrm{~s}$ & $1.35 \mathrm{~s}$ & $2.08 \mathrm{~s}$ \\
$f$ & $-1.54824 \mathrm{e}-01$ & $-1.54984 \mathrm{e}-01$ & $-1.55042 \mathrm{e}-01$ \\
$c$ violation & $0.00 \mathrm{e}+00$ & $0.00 \mathrm{e}+00$ & $0.00 \mathrm{e}+00$ \\
optimality & $4.14 \mathrm{e}-08$ & $2.62 \mathrm{e}-07$ & $8.41 \mathrm{e}-08$ \\
\hline MINOS & $76.08 \mathrm{~s}$ & $203.49 \mathrm{~s}$ & $387.45 \mathrm{~s}$ \\
$f$ & $-1.54824 \mathrm{e}-01$ & $-1.54984 \mathrm{e}-01$ & $-1.55042 \mathrm{e}-01$ \\
$c$ violation & $0.00 \mathrm{e}+00$ & $0.00 \mathrm{e}+00$ & $0.00 \mathrm{e}+00$ \\
optimality & $1.48 \mathrm{e}-07$ & $2.09 \mathrm{e}-07$ & $1.83 \mathrm{e}-07$ \\
\hline SNOPT & $10.25 \mathrm{~s}$ & $31.01 \mathrm{~s}$ & $68.29 \mathrm{~s}$ \\
$f$ & $-1.54824 \mathrm{e}-01$ & $-1.54984 \mathrm{e}-01$ & $-1.55042 \mathrm{e}-01$ \\
$c$ violation & $0.00 \mathrm{e}+00$ & $0.00 \mathrm{e}+00$ & $0.00 \mathrm{e}+00$ \\
optimality & $1.12 \mathrm{e}-14$ & $7.05 \mathrm{e}-15$ & $8.42 \mathrm{e}-15$ \\
\hline
\end{tabular}

$\dagger$ Errors. $\ddagger$ Timed out. $\uparrow$ Failed benchmark tests.

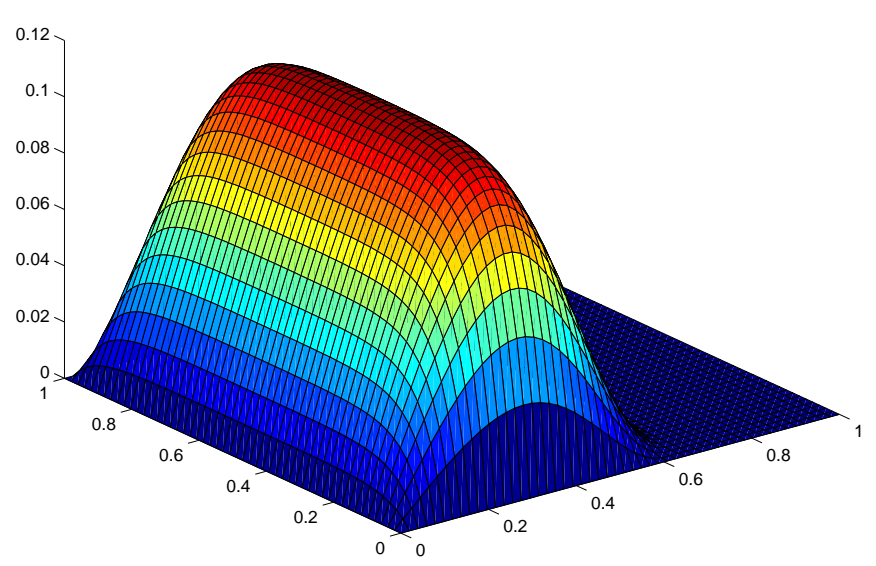

Figure 16.1: Journal bearing problem with $\epsilon=0.1$ 


\section{Minimal Surface with Obstacle}

Find the surface with minimal area that lies above an obstacle with given boundary conditions.

\section{Formulation}

Plateau's problem is to determine the surface of minimal area with a given closed curve in $\mathbb{R}^{3}$ as boundary. We assume that the surface can be represented in nonparametric form $v: \mathbb{R}^{2} \mapsto \mathbb{R}$, and we add the requirement that $v \geq v_{L}$ for some obstacle $v_{L}$. The solution of this obstacle problem [15] minimizes the function $f: K \mapsto \mathbb{R}$,

$$
f(v)=\int_{\mathcal{D}}\left(1+\|\nabla v(x)\|^{2}\right)^{1 / 2} d x
$$

over the convex set $K$, where

$$
K=\left\{v \in H^{1}(\mathcal{D}): v(x)=v_{D}(x) \text { for } x \in \partial \mathcal{D}, \quad v(x) \geq v_{L}(x) \text { for } x \in \mathcal{D}\right\},
$$

$H^{1}(\mathcal{D})$ is the space of functions with gradients in $L^{2}(\mathcal{D})$, the function $v_{D}: \partial \mathcal{D} \mapsto \mathbb{R}$ defines the boundary data, and $v_{L}: \mathcal{D} \mapsto \mathbb{R}$ is the obstacle. We assume that $v_{L} \leq v_{D}$ on the boundary $\partial \mathcal{D}$.

A finite element approximation to the minimal surface problem is obtained by triangulating $\mathcal{D}$ and minimizing $f$ over the space of piecewise linear functions with values $v_{i, j}$ at the vertices of the triangulation. We set $\mathcal{D}=[0,1] \times[0,1]$ and use a triangulation with, respectively, $n_{x}$ and $n_{y}$ internal grid points in the coordinate directions. Data for this problem appears in Table 16.1 .

Table 17.1: Minimal surface problem data

\begin{tabular}{|l|c|}
\hline Variables & $n_{x} n_{y}$ \\
Constraints & 0 \\
Bounds & $n_{x} n_{y}$ \\
Linear equality constraints & 0 \\
Linear inequality constraints & 0 \\
Nonlinear equality constraints & 0 \\
Nonlinear inequality constraints & 0 \\
Nonzeros in $\nabla^{2} f(x)$ & $7 n_{x} n_{y}-4\left(n_{x}+n_{y}\right)+2$ \\
Nonzeros in $c^{\prime}(x)$ & 0 \\
\hline
\end{tabular}

\section{Performance}

We provide results for the AMPL formulation in Table 17.2. For these results we fix $n_{x}=50$ and vary $n_{y}$. The starting guess is the function $1-(2 x-1)^{2}$ evaluated at the grid nodes. We used boundary data

$$
v_{D}(x, y)=\left\{\begin{aligned}
1-(2 x-1)^{2}, & y=0,1 \\
0, & \text { otherwise }
\end{aligned}\right.
$$


and the obstacle

$$
v_{L}(x, y)=\left\{\begin{array}{cl}
1 & \text { if }\left|x-\frac{1}{2}\right| \leq \frac{1}{4},\left|y-\frac{1}{2}\right| \leq \frac{1}{4} \\
0, & \text { otherwise. }
\end{array}\right.
$$

Figure 17.1 shows the minimal surface for this data.

Table 17.2: Performance on minimal surface area with obstacle problem

\begin{tabular}{|c|c|c|c|}
\hline Solver & $n_{y}=50$ & $n_{y}=75$ & $n_{y}=100$ \\
\hline \hline FILTER & $77.67 \mathrm{~s}$ & $244.35 \mathrm{~s}$ & $423.62 \mathrm{~s}$ \\
$f$ & $2.51488 \mathrm{e}+00$ & $2.50568 \mathrm{e}+00$ & $2.50694 \mathrm{e}+00$ \\
$c$ violation & $0.00 \mathrm{e}+00$ & $0.00 \mathrm{e}+00$ & $0.00 \mathrm{e}+00$ \\
optimality & $2.78 \mathrm{e}-07$ & $2.62 \mathrm{e}-07$ & $3.87 \mathrm{e}-07$ \\
\hline KNITRO & $349.67 \mathrm{~s}$ & $985.87 \mathrm{~s}$ & $\ddagger$ \\
$f$ & $2.51488 \mathrm{e}+00 \dagger$ & $2.50568 \mathrm{e}+00$ & $\ddagger$ \\
$c$ violation & $0.00 \mathrm{e}+00 \dagger$ & $0.00 \mathrm{e}+00$ & $\ddagger$ \\
optimality & $1.27 \mathrm{e}-10 \dagger$ & $3.17 \mathrm{e}-07$ & $\ddagger$ \\
\hline LOQO & $3.99 \mathrm{~s}$ & $6.12 \mathrm{~s}$ & $\ddagger$ \\
$f$ & $2.51488 \mathrm{e}+00$ & $2.50568 \mathrm{e}+00$ & $\ddagger$ \\
$c$ violation & $0.00 \mathrm{e}+00$ & $0.00 \mathrm{e}+00$ & $\ddagger$ \\
optimality & $3.00 \mathrm{e}-07$ & $3.31 \mathrm{e}-12$ & $\ddagger$ \\
\hline MINOS & $77.97 \mathrm{~s}$ & $295.46 \mathrm{~s}$ & $700.88 \mathrm{~s}$ \\
$f$ & $2.51488 \mathrm{e}+00$ & $2.50568 \mathrm{e}+00$ & $2.50694 \mathrm{e}+00$ \\
$c$ violation & $0.00 \mathrm{e}+00$ & $0.00 \mathrm{e}+00$ & $0.00 \mathrm{e}+00$ \\
optimality & $1.98 \mathrm{e}-07$ & $2.10 \mathrm{e}-07$ & $4.65 \mathrm{e}-07$ \\
\hline SNOPT & $1726.62 \mathrm{~s}$ & $\ddagger$ & $\ddagger$ \\
$f$ & $2.51488 \mathrm{e}+00$ & $\ddagger$ & $\ddagger$ \\
$c$ violation & $4.73 \mathrm{e}-08$ & $\ddagger$ & $\ddagger$ \\
optimality & $1.79 \mathrm{e}-07$ & $\ddagger$ & $\ddagger$ \\
\hline
\end{tabular}

$\dagger$ Errors. $\ddagger$ Timed out. $\uparrow$ Failed benchmark tests.

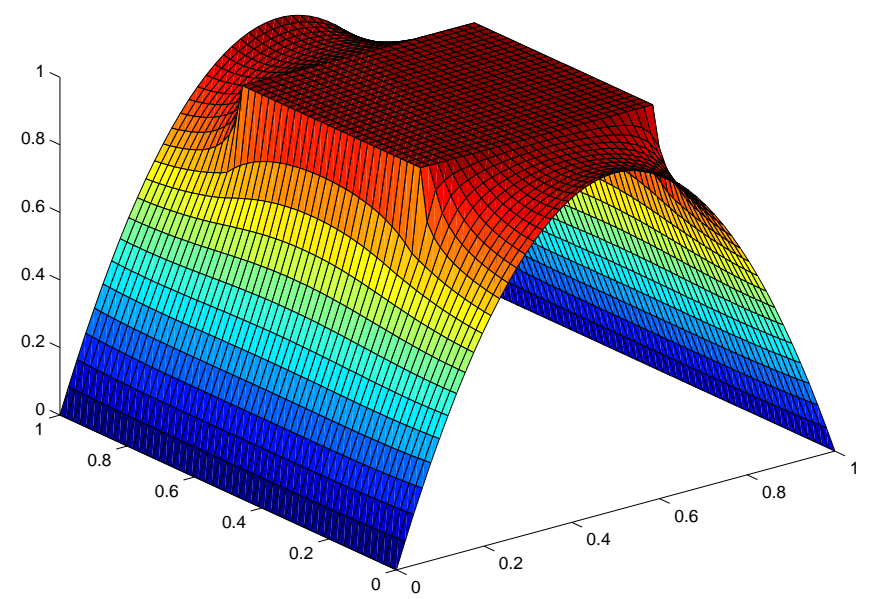

Figure 17.1: Minimal surface problem with a plate obstacle 


\section{Triangular Mesh Smoothing}

Minimize the sum of the inverse weighted mean ratio of the elements in a fixed-boundary triangular mesh by adjusting the locations of the free vertices.

\section{Formulation}

If we define the matrix $A$ to describe the edges of a triangular element with vertices at $\left(x_{0}, y_{0}\right),\left(x_{1}, y_{1}\right)$, and $\left(x_{2}, y_{2}\right)$ as

$$
A=\left(\begin{array}{cc}
x_{1}-x_{0} & x_{2}-x_{0} \\
y_{1}-y_{0} & y_{2}-y_{0}
\end{array}\right)
$$

then the mean ratio metric for the element is

$$
\frac{2 \operatorname{det}(A)}{\|A\|_{F}^{2}}
$$

where $\operatorname{det}(\cdot)$ and $\|\cdot\|_{F}$ denote the determinant and Frobenius norm of the input matrix, respectively. The mean ratio [21] measures the shape-quality of the elements. Weighting the mean ratio metric so that it approaches its maximum value for an equilateral triangle, rather than an isosceles triangle, requires the matrix

$$
W=\left(\begin{array}{cc}
1 & \frac{1}{2} \\
0 & \frac{\sqrt{3}}{2}
\end{array}\right) .
$$

The weighted mean ratio metric is then

$$
\mu=\frac{2 \operatorname{det}\left(A W^{-1}\right)}{\left\|A W^{-1}\right\|_{F}^{2}}=\frac{4 \operatorname{det}(A)}{\sqrt{3}\left\|A W^{-1}\right\|_{F}^{2}},
$$

which is equivalent to the inverse condition number of $A W^{-1}$ [20]. The weighted mean ratio value is 1 for an equilateral triangle and approaches zero as the vertices of the triangle become collinear with at least one nonzero edge length. We minimize

$$
\sum_{k=1}^{e} \mu_{k}^{-1} \text { subject to } \operatorname{det}\left(A_{k}\right) \geq \tau,
$$

where $e$ is the number of elements, $A_{k}$ is the edge matrix for element $k$, and $\tau>0$ is a tolerance based on the minimum element area for the feasible initial point provided. The constraints ensure that the area of each element be greater than $\tau$ and that the elements cannot become inverted, causing folds in the mesh. The objective function is bounded below and twice continuously differentiable on an open set containing the feasible region, but outside of the feasible region the objective can approach $-\infty$.

Approximate data for this problem appears in Table 18.1, where $n$ is the number of non-fixed nodes in the mesh, $e$ is the total number of elements, and $e_{b}$ is the number of elements containing exactly one non-fixed node (boundary elements). The numbers of 
actual nonzeros in the Hessian will be significantly fewer, depending on the number of fixed nodes in the problem and how the nodes are connected to form elements.

Table 18.1: Triangular Mesh Smoothing

\begin{tabular}{|l|c|}
\hline Variables & $2 n$ \\
Constraints & $e$ \\
Bounds & 0 \\
Linear equality constraints & 0 \\
Linear inequality constraints & $e_{b}$ \\
Nonlinear equality constraints & 0 \\
Nonlinear inequality constraints & $e-e_{b}$ \\
Nonzeros in $\nabla^{2} f(x)$ & $36 e-27 e_{b}$ \\
Nonzeros in $c^{\prime}(x)$ & $6 e-4 e_{b}$ \\
\hline
\end{tabular}

\section{Performance}

We provide results for the AMPL formulation in Figure 18.2 for several test problems. The final smoothed mesh for each example is given in Figure 18.1.

Table 18.2: Performance on triangular mesh smoothing problem

\begin{tabular}{|c|c|c|c|}
\hline Solver & deer & pacman & turtle \\
\hline $\begin{array}{l}\text { FILTER } \\
\end{array}$ & $29.76 \mathrm{~s}$ & $11.23 \mathrm{~s}$ & $136.67 \mathrm{~s}$ \\
\hline$f$ & $2.01174 \mathrm{e}+03 \uparrow$ & $1.25045 \mathrm{e}+03 \uparrow$ & $4.21523 \mathrm{e}+03 \uparrow$ \\
\hline$c$ violation & $0.00 \mathrm{e}+00 \uparrow$ & $0.00 \mathrm{e}+00 \uparrow$ & $0.00 \mathrm{e}+00 \uparrow$ \\
\hline optimality & $3.07 \mathrm{e}-06 \uparrow$ & $1.73 \mathrm{e}-06 \uparrow$ & $9.48 \mathrm{e}-06 \uparrow$ \\
\hline KNITRO & $1.24 \mathrm{~s}$ & $0.73 \mathrm{~s}$ & $4.12 \mathrm{~s}$ \\
\hline$f$ & $2.01174 \mathrm{e}+03$ & $1.25045 \mathrm{e}+03$ & $4.21523 \mathrm{e}+03$ \\
\hline$c$ violation & $0.00 \mathrm{e}+00$ & $0.00 \mathrm{e}+00$ & $0.00 \mathrm{e}+00$ \\
\hline optimality & $8.11 \mathrm{e}-07$ & $3.73 \mathrm{e}-07$ & $3.28 \mathrm{e}-07$ \\
\hline$\overline{\mathrm{LOQO}}$ & $t$ & $t$ & 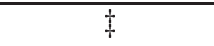 \\
\hline$f$ & $\ddagger$ & 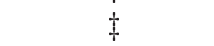 & $\ddagger$ \\
\hline$c$ violation & + & 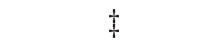 & + \\
\hline optimality & + & $t$ & $t$ \\
\hline MINOS & $29.58 \mathrm{~s}$ & $2.21 \mathrm{~s}$ & $106.04 \mathrm{~s}$ \\
\hline$f$ & $\dagger$ & $-9.73735 \mathrm{e}+11 \dagger$ & $\dagger$ \\
\hline$c$ violation & $\dagger$ & $2.10 \mathrm{e}-03 \dagger$ & $\dagger$ \\
\hline optimality & $\dagger$ & $1.00 \mathrm{e}+00 \dagger$ & $\dagger$ \\
\hline SNOPT & $709.2 \mathrm{~s}$ & $162.06 \mathrm{~s}$ & $38.7 \mathrm{~s}$ \\
\hline$f$ & $2.01174 \mathrm{e}+03$ & $1.25045 \mathrm{e}+03$ & $-1.38468 \mathrm{e}+13 \dagger$ \\
\hline$c$ violation & $0.00 \mathrm{e}+00$ & $0.00 \mathrm{e}+00$ & $2.85 \mathrm{e}-01 \dagger$ \\
\hline optimality & $1.77 \mathrm{e}-07$ & $1.97 \mathrm{e}-07$ & $1.00 \mathrm{e}+00 \dagger$ \\
\hline
\end{tabular}



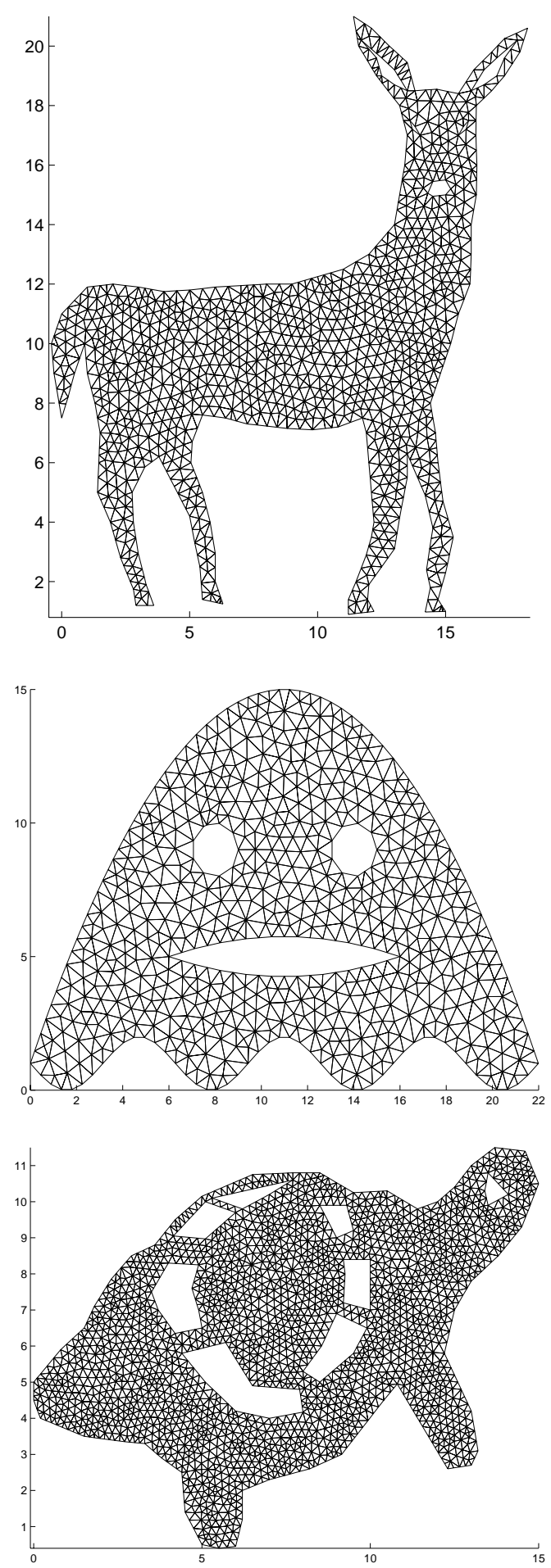

Figure 18.1: Smoothed meshes for the deer, pacman ghost, and turtle 


\section{Tetrahedral Mesh Smoothing}

Minimize the sum of the inverse weighted mean ratio of the elements in a fixed-boundary tetrahedral mesh by adjusting the locations of the free vertices.

\section{Formulation}

If we define the matrix $A$, similar to the matrix used for triangular elements, to describe the edges of a tetrahedral element as

$$
A=\left(\begin{array}{ccc}
x_{1}-x_{0} & x_{2}-x_{0} & x_{3}-x_{0} \\
y_{1}-y_{0} & y_{2}-y_{0} & y_{3}-y_{0} \\
z_{1}-z_{0} & z_{2}-z_{0} & z_{3}-z_{0}
\end{array}\right),
$$

then the mean ratio metric for the element is

$$
\frac{3 \operatorname{det}(A)^{\frac{2}{3}}}{\|A\|_{F}^{2}}
$$

where $\operatorname{det}(\cdot)$ and $\|\cdot\|_{F}$ denote the determinant and Frobenius norm of the input matrix, respectively. The mean ratio [21] measures the shape-quality of the elements. Weighting the mean ratio metric so that it approaches its maximum value for an equilateral tetrahedron, rather than a right-angled tetrahedron, requires the matrix

$$
W=\left(\begin{array}{ccc}
1 & \frac{1}{2} & \frac{1}{2} \\
0 & \frac{\sqrt{3}}{2} & \frac{\sqrt{3}}{6} \\
0 & 0 & \frac{\sqrt{2}}{\sqrt{3}}
\end{array}\right)
$$

The weighted mean ratio metric is then

$$
\mu=\frac{3 \operatorname{det}\left(A W^{-1}\right)^{\frac{2}{3}}}{\left\|A W^{-1}\right\|_{F}^{2}}=\frac{3 \operatorname{det}(A)^{\frac{2}{3}}}{2^{\frac{1}{3}}\left\|A W^{-1}\right\|_{F}^{2}}
$$

This measure is 1 for an equilateral tetrahedron and approaches zero as the vertices of the tetrahedron become coplanar with at least one nonzero edge length. We minimize

$$
\sum_{k=1}^{e} \mu_{k}^{-1} \text { subject to } \operatorname{det}\left(A_{k}\right) \geq \tau,
$$

where $e$ is the number of elements, $A_{k}$ is the edge matrix for element $k$, and $\tau$ is a tolerance based on the minimum element volume for the feasible initial point provided. The constraints ensure that the volume of each element be greater than $\tau$ and that the elements cannot become inverted, causing folds in the mesh. The objective function is bounded below and twice continuously differentiable on an open set containing the feasible region. 
Table 19.1: Tetrahedral Mesh Smoothing

\begin{tabular}{|l|c|}
\hline Variables & $3 n$ \\
Constraints & $e$ \\
Bounds & 0 \\
Linear equality constraints & 0 \\
Linear inequality constraints & $e_{b}$ \\
Nonlinear equality constraints & 0 \\
Nonlinear inequality constraints & $e-e_{b}$ \\
Nonzeros in $\nabla^{2} f(x)$ & $144 e-135 e_{b}$ \\
Nonzeros in $c^{\prime}(x)$ & $9 e-6 e_{b}$ \\
\hline
\end{tabular}

Approximate data for this problem appears in Table 19.1, where $n$ is the number of non-fixed nodes in the mesh, $e$ is the total number of elements, and $e_{b}$ is the number of elements containing exactly one non-fixed node (boundary elements). The numbers of actual nonzeros in the Hessian will be significantly fewer, depending on the number of fixed nodes in the problem and how the nodes are connected to form elements.

\section{Performance}

We provide results for the AMPL formulation in Figure 19 for several test problems. Pictures of the boundary nodes for the samples meshes appear in Figure 19.1.

Table 19.2: Performance on tetrahedral mesh smoothing problem

\begin{tabular}{|c|c|c|c|c|c|}
\hline Solver & duct15 & duct 20 & foam 5 & gear & hook \\
\hline \hline FILTER & $198.88 \mathrm{~s}$ & $28.19 \mathrm{~s}$ & $14.86 \mathrm{~s}$ & $14.18 \mathrm{~s}$ & $112.6 \mathrm{~s}$ \\
$f$ & $1.04951 \mathrm{e}+04$ & $4.82685 \mathrm{e}+03$ & $6.42560 \mathrm{e}+03 \uparrow$ & $4.15163 \mathrm{e}+03 \uparrow$ & $6.05735 \mathrm{e}+03 \uparrow$ \\
$c$ violation & $0.00 \mathrm{e}+00$ & $0.00 \mathrm{e}+00$ & $0.00 \mathrm{e}+00 \uparrow$ & $0.00 \mathrm{e}+00 \uparrow$ & $0.00 \mathrm{e}+00 \uparrow$ \\
optimality & $4.42 \mathrm{e}-07$ & $2.52 \mathrm{e}-07$ & $9.32 \mathrm{e}-06 \uparrow$ & $1.72 \mathrm{e}-05 \uparrow$ & $1.22 \mathrm{e}-06 \uparrow$ \\
\hline KNITRO & $15.74 \mathrm{~s}$ & $5.85 \mathrm{~s}$ & $4.53 \mathrm{~s}$ & $4.41 \mathrm{~s}$ & $7.21 \mathrm{~s}$ \\
$f$ & $1.04951 \mathrm{e}+04$ & $4.82685 \mathrm{e}+03$ & $6.42560 \mathrm{e}+03$ & $4.15163 \mathrm{e}+03$ & $6.05735 \mathrm{e}+03$ \\
$c$ violation & $0.00 \mathrm{e}+00$ & $0.00 \mathrm{e}+00$ & $0.00 \mathrm{e}+00$ & $0.00 \mathrm{e}+00$ & $0.00 \mathrm{e}+00$ \\
optimality & $7.15 \mathrm{e}-07$ & $5.09 \mathrm{e}-07$ & $6.41 \mathrm{e}-07$ & $8.54 \mathrm{e}-07$ & $1.09 \mathrm{e}-07$ \\
\hline LOQO & $28.39 \mathrm{~s}$ & $10.5 \mathrm{~s}$ & $5.99 \mathrm{~s}$ & $3.86 \mathrm{~s}$ & $8.99 \mathrm{~s}$ \\
$f$ & $1.04951 \mathrm{e}+04$ & $4.82685 \mathrm{e}+03$ & $6.42560 \mathrm{e}+03$ & $4.15163 \mathrm{e}+03$ & $6.05735 \mathrm{e}+03$ \\
$c$ violation & $0.00 \mathrm{e}+00$ & $0.00 \mathrm{e}+00$ & $0.00 \mathrm{e}+00$ & $0.00 \mathrm{e}+00$ & $0.00 \mathrm{e}+00$ \\
optimality & $1.33 \mathrm{e}-08$ & $1.18 \mathrm{e}-08$ & $1.56 \mathrm{e}-07$ & $9.42 \mathrm{e}-08$ & $6.37 \mathrm{e}-08$ \\
\hline MINOS & $199.71 \mathrm{~s}$ & $244.54 \mathrm{~s}$ & $1.48 \mathrm{~s}$ & $1.17 \mathrm{~s}$ & $3.86 \mathrm{~s}$ \\
$f$ & $\dagger$ & $\dagger$ & $\dagger$ & $\dagger$ & $\dagger$ \\
$c$ violation & $\dagger$ & $\dagger$ & $\dagger$ & $\dagger$ & $\dagger$ \\
optimality & $\dagger$ & $\dagger$ & $\dagger$ & $\dagger$ & $\dagger$ \\
\hline SNOPT & $1767.77 \mathrm{~s}$ & $120.82 \mathrm{~s}$ & $118.97 \mathrm{~s}$ & $67.41 \mathrm{~s}$ & $240.23 \mathrm{~s}$ \\
$f$ & $1.04951 \mathrm{e}+04$ & $4.82685 \mathrm{e}+03$ & $6.42560 \mathrm{e}+03$ & $4.15163 \mathrm{e}+03$ & $6.05735 \mathrm{e}+03$ \\
$c$ violation & $0.00 \mathrm{e}+00$ & $0.00 \mathrm{e}+00$ & $0.00 \mathrm{e}+00$ & $0.00 \mathrm{e}+00$ & $0.00 \mathrm{e}+00$ \\
optimality & $1.89 \mathrm{e}-07$ & $8.87 \mathrm{e}-08$ & $1.44 \mathrm{e}-07$ & $1.70 \mathrm{e}-07$ & $1.99 \mathrm{e}-07$ \\
\hline
\end{tabular}

$\dagger$ Errors. $\ddagger$ Timed out. $\uparrow$ Failed benchmark tests. 

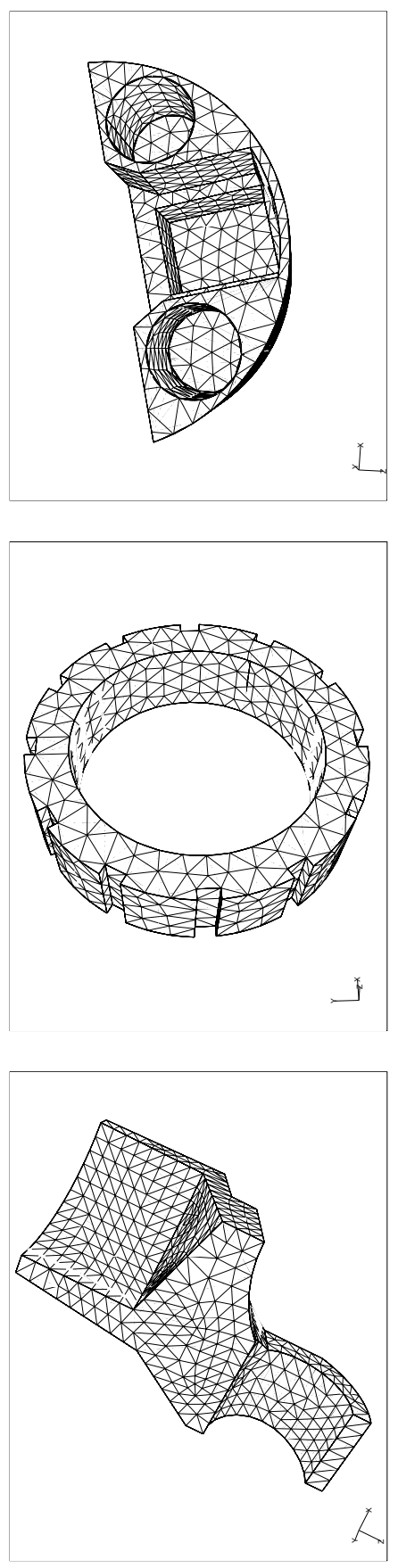

Figure 19.1: Fixed boundary nodes for foam, gear, and hook. 


\section{Transition States for the Lane-Emden Problem}

Determine a transition state (mountain-pass) for the Lane-Emden problem. A transition state is a solution to the Lane-Emden equation for which the energy increases along any $k$-dimensional subspace with $k>1$.

\section{Formulation}

The Lane-Emden problem is defined by the functional $f: H_{0}^{1}(\mathcal{D}) \mapsto \mathbb{R}$,

$$
f(u)=\int_{\mathcal{D}}\left(\frac{1}{2}\|\nabla u(s)\|^{2}-\frac{1}{4} u(s)^{4}\right) d s .
$$

This functional has an infinite number of critical points (Struwe [30, Theorem 6.6]), and every critical point satisfies the Lane-Emden equation

$$
-\Delta u=u^{3}, \quad x \in \mathcal{D} .
$$

In particular, nontrivial critical points $u^{+} \geq 0 \geq u^{-}$exist. Chen, Zhou, and Ni [11] provide additional information on the theoretical properties of the solution to this problem.

A finite element approximation to this integral is obtained by triangulating $\mathcal{D}$ and approximating $f$ over the space of piecewise linear functions with values $u_{i, j}$ at the vertices of the triangulation. For this problem the domain $\mathcal{D}$ is the unit square with a triangulation containing $n$ internal grid points generated by TRIANGLE [28, 29].

The elastic string algorithm [23] finds an approximate mountain pass solution by solving the constrained optimization problem

$$
\min \left\{w: f\left(u_{k}\right) \leq w, 1 \leq k \leq m,\left\|u_{k}-u_{k+1}\right\| \leq h, 0 \leq k \leq m\right\}
$$

where $u_{0}=u_{a}$ and $u_{m+1}=u_{b}$ are fixed points separated by a mountain range and $h>0$ is a fixed tolerance. Data for this problem appears in Table 20.1.

Table 20.1: Lane-Emden problem data

\begin{tabular}{|l|c|}
\hline Variables & $m n+1$ \\
Constraints & $2 m+1$ \\
Bounds & 0 \\
Linear equality constraints & 0 \\
Linear inequality constraints & 0 \\
Nonlinear equality constraints & 0 \\
Nonlinear inequality constraints & $2 m+1$ \\
Nonzeros in $\nabla^{2} f(x)$ & 0 \\
Nonzeros in $c^{\prime}(x)$ & $m(3 n+1)$ \\
\hline
\end{tabular}

\section{Performance}

We provide results for the AMPL formulation in Table 20.2. For these results the discretization was fixed, but the number of internal breakpoints $m$ in the elastic string algorithm was 
varied. The graph on the left of Figure 20.1 is a plot of the path profile drawn from the function values $f\left(u_{k}\right)$ for $0 \leq k \leq m+1$, while the graph on the right is a plot of the mountain pass solution.

Note that the maximum along the path profile is achieved at two points, which is to be expected. The ragged nature of the path profile for the Lane-Emden problem is due to the use of $m=10$; a smoother profile is obtained with higher values of $m$.

Table 20.2: Performance on Lane-Emden problem

\begin{tabular}{|c|c|c|c|}
\hline Solver & 10 & 20 & 40 \\
\hline \hline FILTER & $\ddagger$ & $\ddagger$ & $\ddagger$ \\
$f$ & $\ddagger$ & $\ddagger$ & $\ddagger$ \\
$c$ violation & $\ddagger$ & $\ddagger$ & $\ddagger$ \\
\hline KNITRO & $46.12 \mathrm{~s}$ & $33.42 \mathrm{~s}$ & $81.72 \mathrm{~s}$ \\
$f$ & $8.49464 \mathrm{e}+00$ & $9.11000 \mathrm{e}+00$ & $9.28489 \mathrm{e}+00$ \\
$c$ violation & $0.00 \mathrm{e}+00$ & $0.00 \mathrm{e}+00$ & $0.00 \mathrm{e}+00$ \\
optimality & $6.99 \mathrm{e}-09$ & $6.14 \mathrm{e}-08$ & $1.30 \mathrm{e}-08$ \\
\hline LOQO & $83.46 \mathrm{~s}$ & $173.12 \mathrm{~s}$ & $1372.39 \mathrm{~s}$ \\
$f$ & $8.49464 \mathrm{e}+00$ & $9.11000 \mathrm{e}+00$ & $9.28489 \mathrm{e}+00$ \\
$c$ violation & $4.79 \mathrm{e}-09$ & $5.08 \mathrm{e}-09$ & $7.33 \mathrm{e}-10$ \\
optimality & $2.80 \mathrm{e}-10$ & $9.47 \mathrm{e}-11$ & $9.04 \mathrm{e}-12$ \\
\hline MINOS & $418.71 \mathrm{~s}$ & $\ddagger$ & $\ddagger$ \\
$f$ & $8.49464 \mathrm{e}+00$ & $\ddagger$ & $\ddagger$ \\
$c$ violation & $9.19 \mathrm{e}-12$ & $\ddagger$ & $\ddagger$ \\
optimality & $2.51 \mathrm{e}-07$ & $\ddagger$ & $\ddagger$ \\
\hline SNOPT & $1177.98 \mathrm{~s}$ & $\ddagger$ & $\ddagger$ \\
$f$ & $8.49464 \mathrm{e}+00$ & $\ddagger$ & $\ddagger$ \\
$c$ violation & $7.17 \mathrm{e}-13$ & $\ddagger$ & $\ddagger$ \\
optimality & $1.81 \mathrm{e}-07$ & $\ddagger$ & $\ddagger$ \\
\hline
\end{tabular}

$\dagger$ Errors. + Timed out. $\uparrow$ Failed benchmark tests.
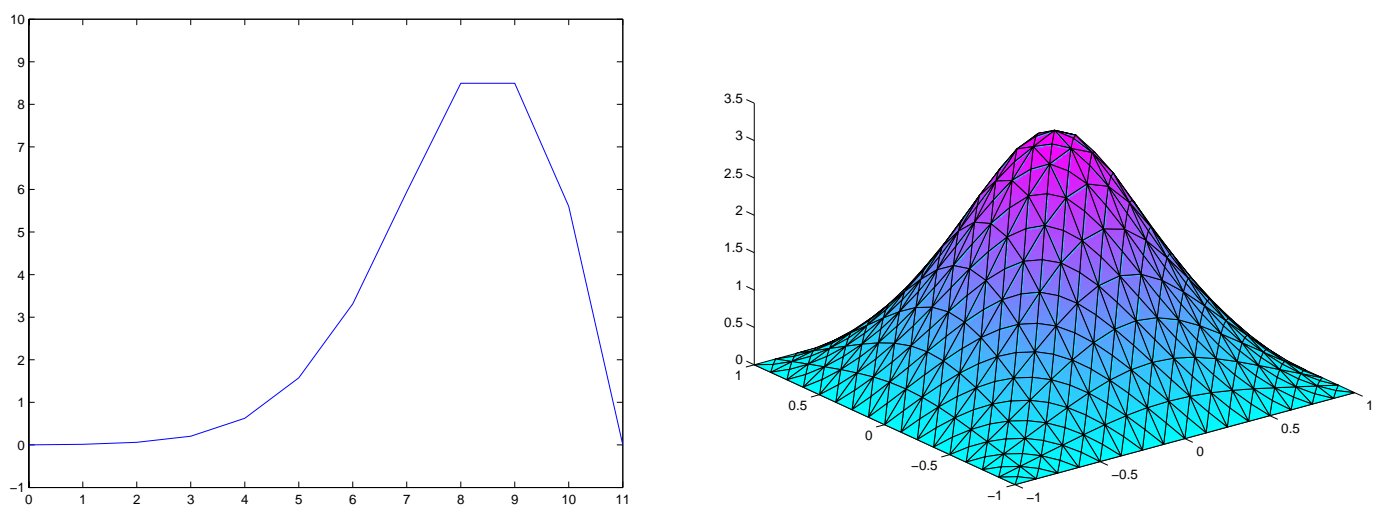

Figure 20.1: Path profile and mountain pass for the Lane-Emden problem. 


\section{Transition States for the Dirichlet Problem}

Determine a transition state (mountain-pass) for the (singularly perturbed) Dirichlet problem. A transition state is a solution to the Dirichlet equation for which the energy increases along any $k$-dimensional subspace with $k>1$.

\section{Formulation}

The (singularly perturbed) Dirichlet problem is defined by the functional $f: H_{0}^{1}(\mathcal{D}) \mapsto \mathbb{R}$,

$$
f(u)=\int_{\mathcal{D}}\left(\frac{\varepsilon^{2}}{2}\|\nabla u(s)\|^{2}+\frac{1}{2} u(s)^{2}-\frac{1}{4} u(s)^{4}\right) d s,
$$

where $\varepsilon \in(0,1)$. A finite element approximation to this integral is obtained by triangulating $\mathcal{D}$ and approximating $f$ over the space of piecewise linear functions with values $u_{i, j}$ at the vertices of the triangulation. For this problem the domain $\mathcal{D}$ is the unit circle with a triangulation containing $n$ internal grid points generated by TRIANGLE [28, 29].

The elastic string algorithm [23] finds an approximate mountain pass solution by solving the constrained optimization problem

$$
\min \left\{w: f\left(u_{k}\right) \leq w, 1 \leq k \leq m, \quad\left\|u_{k}-u_{k+1}\right\| \leq h, 0 \leq k \leq m\right\}
$$

where $u_{0}=u_{a}$ and $u_{m+1}=u_{b}$ are fixed points separated by a mountain range and $h>0$ is a fixed tolerance. Data for this problem appears in Table 21.1.

Table 21.1: Dirichlet problem data

\begin{tabular}{|l|c|}
\hline Variables & $m n+1$ \\
Constraints & $2 m+1$ \\
Bounds & 0 \\
Linear equality constraints & 0 \\
Linear inequality constraints & 0 \\
Nonlinear equality constraints & 0 \\
Nonlinear inequality constraints & $2 m+1$ \\
Nonzeros in $\nabla^{2} f(x)$ & 0 \\
Nonzeros in $c^{\prime}(x)$ & $m(3 n+1)$ \\
\hline
\end{tabular}

\section{Performance}

We provide results for the AMPL formulation in Table 21.2 when $\varepsilon=0.1$. For these results the discretization was fixed, but the number of internal breakpoints $m$ in the elastic string algorithm was varied. The graph on the left of Figure 21.1 is a plot of the path profile drawn from the function values $f\left(u_{k}\right)$ for $0 \leq k \leq m+1$, while the graph on the right is a plot of the mountain pass solution. 
Table 21.2: Performance on perturbed Dirichlet problem

\begin{tabular}{|c|c|c|c|}
\hline Solver & 10 & 20 & 40 \\
\hline \hline FILTER & $710.39 \mathrm{~s}$ & $1272.75 \mathrm{~s}$ & $\ddagger$ \\
$f$ & $9.33495 \mathrm{e}-04$ & $2.05683 \mathrm{e}-02$ & $\ddagger$ \\
$c$ violation & $3.44 \mathrm{e}-13$ & $3.50 \mathrm{e}-12$ & $\ddagger$ \\
optimality & $4.45 \mathrm{e}-08$ & $5.35 \mathrm{e}-08$ & $\ddagger$ \\
\hline KNITRO & $1614.05 \mathrm{~s}$ & $145.8 \mathrm{~s}$ & $329.15 \mathrm{~s}$ \\
$f$ & $1.93590 \mathrm{e}-06$ & $1.71467 \mathrm{e}-02$ & $3.28852 \mathrm{e}-02$ \\
$c$ violation & $0.00 \mathrm{e}+00$ & $0.00 \mathrm{e}+00$ & $0.00 \mathrm{e}+00$ \\
optimality & $5.78 \mathrm{e}-07$ & $4.20 \mathrm{e}-08$ & $1.23 \mathrm{e}-07$ \\
\hline LOQO & $259.51 \mathrm{~s}$ & $643.59 \mathrm{~s}$ & $1276.72 \mathrm{~s}$ \\
$f$ & $9.33494 \mathrm{e}-04$ & $1.71464 \mathrm{e}-02$ & $3.01527 \mathrm{e}-02$ \\
$c$ violation & $9.40 \mathrm{e}-09$ & $2.17 \mathrm{e}-08$ & $6.77 \mathrm{e}-08$ \\
optimality & $1.58 \mathrm{e}-09$ & $2.32 \mathrm{e}-10$ & $1.33 \mathrm{e}-10$ \\
\hline MINOS & $512.63 \mathrm{~s}$ & $28.16 \mathrm{~s}$ & $54.48 \mathrm{~s}$ \\
$f$ & $2.82702 \mathrm{e}-12$ & $1.62658 \mathrm{e}+04 \dagger$ & $6.21175 \mathrm{e}+04 \dagger$ \\
$c$ violation & $4.85 \mathrm{e}-10$ & $1.00 \mathrm{e}+00 \dagger$ & $1.00 \mathrm{e}+00 \dagger$ \\
optimality & $4.80 \mathrm{e}-08$ & $1.00 \mathrm{e}+00 \dagger$ & $1.00 \mathrm{e}+00 \dagger$ \\
\hline SNOPT & $\ddagger$ & $\ddagger$ & $\ddagger$ \\
$f$ & $\ddagger$ & $\ddagger$ & $\ddagger$ \\
$c$ violation & $\ddagger$ & $\ddagger$ & $\ddagger$ \\
optimality & $\ddagger$ & $\ddagger$ & $\ddagger$ \\
\hline
\end{tabular}

$\dagger$ Errors. $\ddagger$ Timed out. $\uparrow$ Failed benchmark tests.
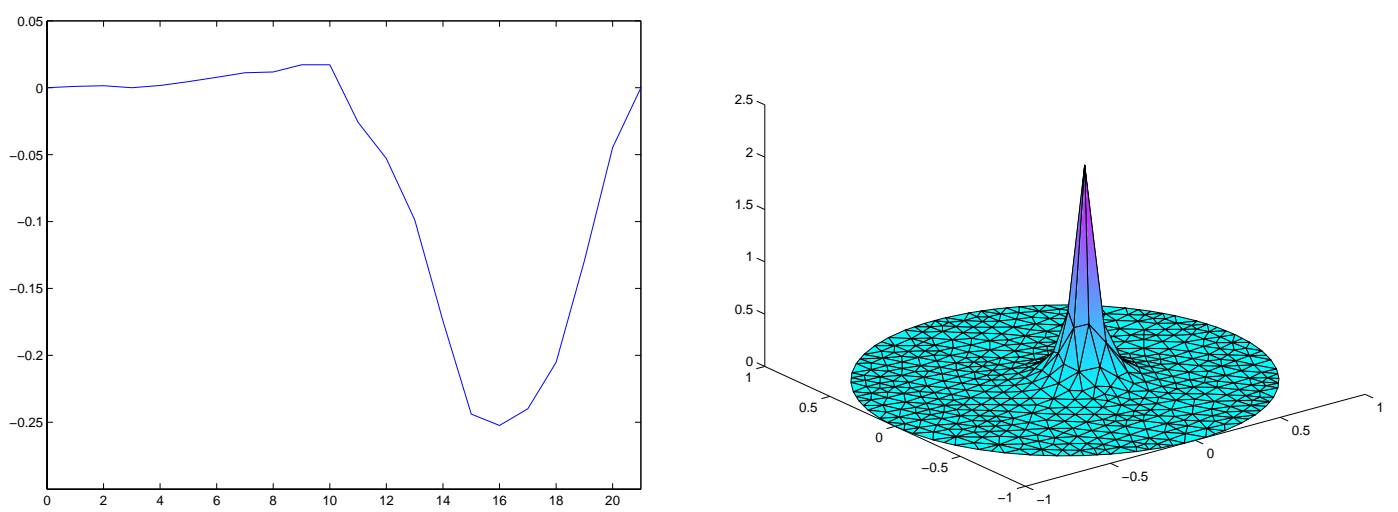

Figure 21.1: Path profile and mountain pass for the singularly perturbed Dirichlet problem. 


\section{Transition States for the Henon Problem}

Determine a transition state (mountain-pass) for the Henon problem. A transition state is a solution to the Henon equation for which the energy increases along any $k$-dimensional subspace with $k>1$.

\section{Formulation}

The Henon problem is defined by the functional $f: H_{0}^{1}(\mathcal{D}) \mapsto \mathbb{R}$,

$$
f(u)=\int_{\mathcal{D}}\left(\frac{1}{2}\|\nabla u(s)\|^{2}-\frac{\|s\|}{4} u(s)^{4}\right) d s .
$$

A finite element approximation to this integral is obtained by triangulating $\mathcal{D}$ and approximating $f$ over the space of piecewise linear functions with values $u_{i, j}$ at the vertices of the triangulation. For this problem the domain $\mathcal{D}$ is the unit circle with a half unit square cut from the center. A triangulation of this domain containing $n$ internal grid points generated by TRIANGLE [28, 29].

The elastic string algorithm [23] finds an approximate mountain pass solution by solving the constrained optimization problem

$$
\min \left\{w: f\left(u_{k}\right) \leq w, 1 \leq k \leq m,\left\|u_{k}-u_{k+1}\right\| \leq h, 0 \leq k \leq m\right\}
$$

where $u_{0}=u_{a}$ and $u_{m+1}=u_{b}$ are fixed points separated by a mountain range and $h>0$ is a fixed tolerance. Data for this problem appears in Table 22.1.

Table 22.1: Henon problem data

\begin{tabular}{|l|c|}
\hline Variables & $m n+1$ \\
Constraints & $2 m+1$ \\
Bounds & 0 \\
Linear equality constraints & 0 \\
Linear inequality constraints & 0 \\
Nonlinear equality constraints & 0 \\
Nonlinear inequality constraints & $2 m+1$ \\
Nonzeros in $\nabla^{2} f(x)$ & 0 \\
Nonzeros in $c^{\prime}(x)$ & $m(3 n+1)$ \\
\hline
\end{tabular}

\section{Performance}

We provide results for the AMPL formulation in Table 22.2. For these results the discretization was fixed, but the number of internal breakpoints $m$ in the elastic string algorithm was varied. The graph on the left of Figure 22.1 is a plot of the path profile drawn from the function values $f\left(u_{k}\right)$ for $0 \leq k \leq m+1$, while the graph on the right is a plot of the mountain pass solution.

The plot of the mountain pass in Figure 22.1 shows that the solution lacks symmetry. In general, the symmetry properties of the domain $\mathcal{D}$ are reflected in the symmetry properties 
of the solution. For example, Chen, Zhou, and Ni [11] noted that on an annular domain there is a solution that is not rotationally symmetric; and since the domain is rotationally symmetric, the mountain-pass solutions form a connected nontrivial set. In particular, the mountain-pass solutions are not isolated. For our domain, which is not rotationally symmetric, there seem to be four distinct mountain-pass solutions.

Table 22.2: Performance on Henon problem

\begin{tabular}{|c|c|c|c|}
\hline Solver & 10 & 20 & 40 \\
\hline \hline FILTER & $1360.44 \mathrm{~s}$ & $566.42 \mathrm{~s}$ & $1666.75 \mathrm{~s}$ \\
$f$ & $7.21915 \mathrm{e}+00$ & $9.50782 \mathrm{e}+01$ & $1.25971 \mathrm{e}+02$ \\
$c$ violation & $2.18 \mathrm{e}-12$ & $4.71 \mathrm{e}-12$ & $3.32 \mathrm{e}-11$ \\
optimality & $2.87 \mathrm{e}-07$ & $2.78 \mathrm{e}-07$ & $2.36 \mathrm{e}-07$ \\
\hline KNITRO & $29.26 \mathrm{~s}$ & $70 \mathrm{~s}$ & $160.69 \mathrm{~s}$ \\
$f$ & $1.93254 \mathrm{e}+01$ & $9.50782 \mathrm{e}+01$ & $1.25971 \mathrm{e}+02$ \\
$c$ violation & $0.00 \mathrm{e}+00$ & $0.00 \mathrm{e}+00$ & $0.00 \mathrm{e}+00$ \\
optimality & $1.58 \mathrm{e}-09$ & $5.51 \mathrm{e}-08$ & $1.55 \mathrm{e}-07$ \\
\hline LOQO & $101.01 \mathrm{~s}$ & $209.9 \mathrm{~s}$ & $537.75 \mathrm{~s}$ \\
$f$ & $4.26810 \mathrm{e}+00$ & $9.24570 \mathrm{e}+01$ & $1.25971 \mathrm{e}+02$ \\
$c$ violation & $2.72 \mathrm{e}-08$ & $6.82 \mathrm{e}-09$ & $1.10 \mathrm{e}-08$ \\
optimality & $1.02 \mathrm{e}-10$ & $1.97 \mathrm{e}-11$ & $4.67 \mathrm{e}-11$ \\
\hline MINOS & $1092.05 \mathrm{~s}$ & $1371.3 \mathrm{~s}$ & $\ddagger$ \\
$f$ & $7.21915 \mathrm{e}+00$ & $7.52141 \mathrm{e}+01$ & $\ddagger$ \\
$c$ violation & $2.79 \mathrm{e}-09$ & $2.41 \mathrm{e}-10$ & $\ddagger$ \\
optimality & $2.39 \mathrm{e}-07$ & $2.12 \mathrm{e}-07$ & $\ddagger$ \\
\hline SNOPT & $\ddagger$ & $\ddagger$ & $\ddagger$ \\
$f$ & $\ddagger$ & $\ddagger$ & $\ddagger$ \\
$c$ violation & $\ddagger$ & $\ddagger$ & $\ddagger$ \\
optimality & $\ddagger$ & $\ddagger$ & $\ddagger$ \\
\hline
\end{tabular}

$\dagger$ Errors. $\ddagger$ Timed out. $\uparrow$ Failed benchmark tests.
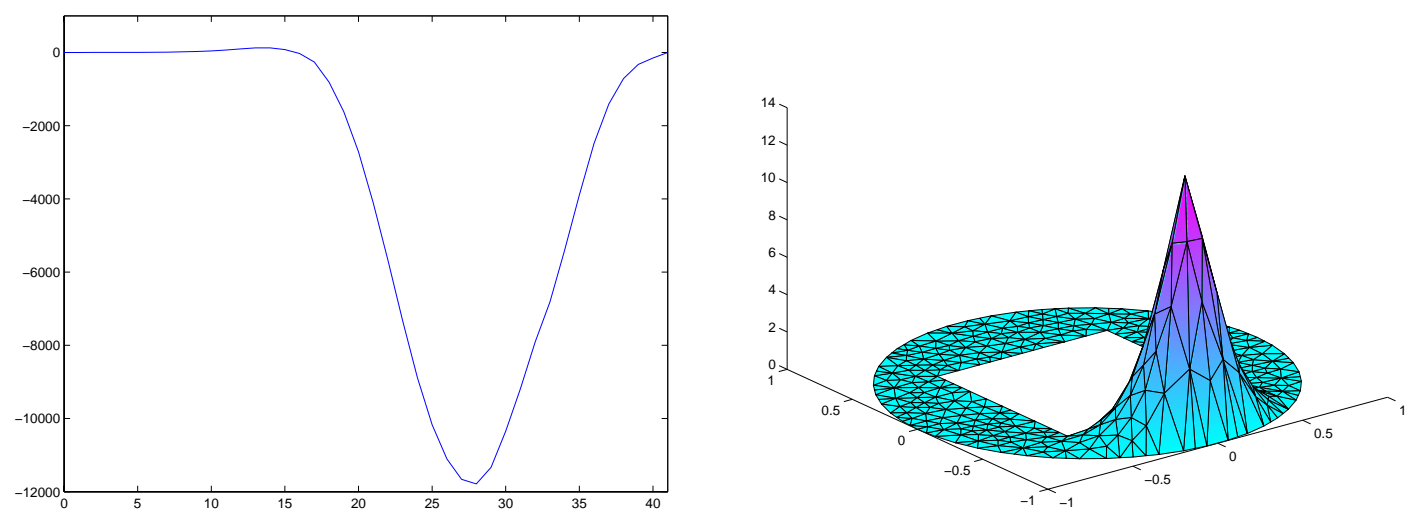

Figure 22.1: Path profile and mountain pass for the Henon equation. 


\section{References}

[1] M. Anitescu And R. Serban, A sparse superlinearly convergent SQP with applications to two-dimensional shape optimization, Preprint ANL/MCS-P706-0198, Argonne National Laboratory, Argonne, Illinois, 1998.

[2] U. M. Ascher, R. M. M. MattheiJ, And R. D. Russell, Numerical solution of boundary value problems for ordinary differential equations, SIAM, 1995.

[3] B. M. Averick, R. G. Carter, J. J. Moré, and G.-L. Xue, The MiNPACK-2 test problem collection, Preprint MCS-P153-0694, Mathematics and Computer Science Division, Argonne National Laboratory, Argonne, Illinois, 1994.

[4] J. Betts, S. Eldersveld, And W. Huffman, Sparse nonlinear programming test problems (Release 1.0), Technical report BCSTECH-93-047, Boeing Computer Services, Seattle, Washington, 1993.

[5] G. E. P. Box, W. G. Hunter, J. F. MacGregor, and J. Erjavec, Some problems associated with the analysis of multiresponse data, Technometrics, 15 (1973), pp. 33-51.

[6] A. Bryson And Y. Ho, Applied Optimal Control: Optimization, Estimation, and Control, John Wiley \& Sons, 1975.

[7] A. E. Bryson, Dynamic Optimization, Addison-Wesley, 1999.

[8] R. Bulinsch, E. Nerz, H. J. Pesch, and O. von Stryk, Combining direct and indirect methods in nonlinear optimal control: Range maximization of a hang glider, in Optimal Control, R. Bulirsch, A. Miele, J. Stoer, and K. H. Well, eds., Birkhäuser Verlag, 1993, pp. 273-288.

[9] G. Capriz and G. Cimatti, Free boundary problems in the theory of hydrodynamic lubrication: A survey, in Free Boundary Problems: Theory and Applications, A. Fasano and M. Primicerio, eds., no. 79 in Research Notes in Mathematics, Pitman, 1983, pp. 613-635.

[10] L. Cesari, Optimization - Theory and Applications, Springer Verlag, 1983.

[11] G. Chen, J. Zhou, And W. Ni, Algorithms and visualization for solutions of nonlinear elliptic equations, Internat. J. Bifur. Chaos, 10 (2000), pp. 1565-1612.

[12] COPS. See www.mcs.anl.gov/ ${ }^{\sim m o r e / c o p s . ~}$

[13] R. Fletcher and S. LeyfFer, User manual for filterSQP, Report NA/181, University of Dundee, 1998.

[14] C. A. Floudas, P. M. Pardalos, C. S. Adjiman, W. R. Esposito, Z. H. Gumus, S. T. Harding, J. L. Klepeis, C. A. Meyer, And C. A. Schweiger, Handbook of Test Problems for Local and Global Optimization, Kluwer Academic Publishers, 1999. 
[15] A. Friedman, Free boundary problems in science and technology, Notices Amer. Math. Soc., 47 (2000), pp. 854-861.

[16] D. GAY, AMPL models. See www.netlib.org/ampl/models.

[17] P. E. Gill, W. Murray, and M. A. Saunders, User's guide for SNOPT 5.3: A Fortran package for large-scale nonlinear programming, Report NA97-5, University of California, San Diego, 1997.

[18] R. Glowinski, Numerical Methods for Nonlinear Variational Problems, SpringerVerlag, 1984.

[19] R. L. Graham, The largest small hexagon, J. Combin. Th., 18 (1975), pp. 165-170.

[20] P. KnupP, Algebraic mesh quality metrics, SIAM J. Sci. Comput., 23(1) (2001), pp. 193-218.

[21] A. Liu AND B. Joe, Relationship between tetrahedron quality measures, BIT, 34 (1994), pp. 268-287.

[22] G. MARIA, An adaptive strategy for solving kinetic model concomitant estimation reduction problems, Can. J. Chem. Eng., 67 (1989), p. 825.

[23] J. J. Moré And T. S. Munson, Computing mountain passes, Preprint ANL/MCSP957-0502, Argonne National Laboratory, Argonne, Illinois, 2002.

[24] J. R. Morris, D. M. Deaven, And K. M. Ho, Genetic algorithm energy minimization for point charges on a sphere, Phys. Rev. B, 53 (1996), pp. R1740-R1743.

[25] B. A. Murtagh and M. A. Saunders, MiNOS 5.5 user's guide, Report SOL 83-20R, Stanford University, 1983, revised July 1998.

[26] B. J. Rothschild, A. F. Sharov, A. J. Kearsley, and A. S. Bondarenko, Estimating growth and mortality in stage-structured populations, Journal of Plankton Research, 19 (1997), pp. 1913-1928.

[27] E. B. Saff And A. KuiJlaArs, Distributing many points on the sphere, Math. Intelligencer, 19 (1997), pp. 5-11.

[28] J. Shewchuk, Triangle: Engineering a 2D Quality Mesh Generator and Delaunay Triangulator, in Applied Computational Geometry: Towards Geometric Engineering, M. C. Lin and D. Manocha, eds., vol. 1148 of Lecture Notes in Computer Science, Springer-Verlag, May 1996, pp. 203-222. From the First ACM Workshop on Applied Computational Geometry.

[29] — Delaunay refinement algorithms for triangular mesh generation, Computational Geometry: Theory and Applications, 22 (2002), pp. 21-74.

[30] M. Struwe, Variational Methods, Springer-Verlag, 2000. 
[31] I.-B. TJoA AND L. T. BIEGLER, Simultaneous solution and optimization strategies for parameter estimation of differential-algebraic equations systems, Ind. Eng. Chem. Res., 30 (1991), pp. 376-385.

[32] R. J. VANDERBEI, Nonlinear optimization models. See www.sor.princeton.edu/ rvdb/ampl/nlmodels.

[33] — LOQO user's manual - Version 4.05, 2000.

[34] O. VON STRYK, User's guide for DIRCOL (Version 2.1): A direct collocation method for the numerical solution of optimal control problems, technical report, Technische Universität München, 1999.

[35] R. Waltz And J. Nocedal, KNITRO user's manual - Version 3.1, Tech. Rep. 5, Northwestern University, Evanston, 2003. 